\title{
DJ-1 promotes cell proliferation and tumor metastasis in esophageal squamous cell carcinoma via the Wnt/ $\beta$-catenin signaling pathway
}

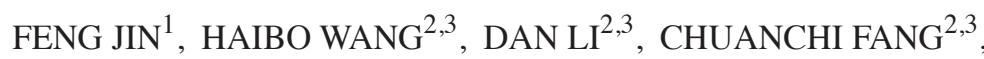 \\ WENYUAN LI ${ }^{2,3}$, QINGTONG SHI ${ }^{4}$, YALI DIAO ${ }^{4}$, ZHIYAN DING $^{5}$, XIAOJUN DAI $^{2,3,6}$, LI TAO $^{2,3}$, \\ MASATAKA SUNAGAWA $^{7}$, FENG WU ${ }^{1}$, YAYUN QIAN ${ }^{2,3}$ and YANQINGLIU ${ }^{2,3}$
}

\begin{abstract}
${ }^{1}$ Department of Respiratory Medicine, The Affiliated Hospital of Yangzhou University, Yangzhou University, Yangzhou, Jiangsu 225001; ${ }^{2}$ Institution of Combining Chinese Traditional and Western Medicine, Medical College;

${ }^{3}$ The State Administration of Traditional Chinese Medicine Key Laboratory of Toxic Pathogens-Based Therapeutic Approaches to Gastric Cancer, Yangzhou University, Yangzhou, Jiangsu 225009; Departments of ${ }^{4}$ Thoracic Surgery and ${ }^{5}$ Pathology, The Affiliated Hospital of Yangzhou University, Yangzhou University, Yangzhou, Jiangsu 225001; ${ }^{6}$ Yangzhou Traditional Chinese Medicine Hospital, Yangzhou, Jiangsu 225009, P.R. China;

${ }^{7}$ Department of Physiology, School of Medicine, Showa University, Tokyo 142-8555, Japan
\end{abstract}

Received January 7, 2019; Accepted January 22, 2020

DOI: 10.3892/ijo.2020.5005

\begin{abstract}
DJ-1, an oncogene, has been reported to be an independent prognostic indicator of poor survival in patients with esophageal squamous cell carcinoma (ESCC). The aim of the present study was to investigate the role of DJ-1 in tumor cell proliferation and invasion in ESCC and its underlying mechanisms. It was observed that the expression level of DJ-1 was upregulated and positively associated with EMT biomarkers in 84 human ESCC tissue specimens. Overexpression and knockdown experiments demonstrated that DJ-1 was involved in proliferation, migration, invasion and EMT in ECA-109 cells in vitro and extensive peritoneal seeding in a peritoneal dissemination mice model. Furthermore, the present data revealed that DJ-1 could activate the Wnt/ $\beta$-catenin signaling pathway, which mediates the EMT and metastasis in ESCC. In conclusions, DJ-1 promoted proliferation, invasion, metastasis and the EMT in ESCC via activation of the Wnt/ $/$-catenin signal pathway. The present results suggested DJ-1 could represent a promising therapeutic target for the prevention and treatment of ESCC-related metastasis.
\end{abstract}

Correspondence to: Professor Yanqing Liu, Institution of Combining Chinese Traditional and Western Medicine, Medical College, Yangzhou University, 136 Jiangyang Middle Road, Yangzhou, Jiangsu 225009, P.R. China

E-mail: yzumpi@163.com

Key words: DJ-1, esophageal squamous cell carcinoma, cell proliferation, invasion, $\mathrm{Wnt} / \beta$-catenin signaling pathway

\section{Introduction}

Esophageal carcinoma is a commonly occurring tumor, with no effective therapy, which threatens human health, causing $>400,000$ mortalities worldwide annually in $2016(1,2)$. The most prevalent type of esophageal carcinoma is esophageal squamous cell carcinoma (ESCC), which mainly occurs in Eastern Asia, as well as Eastern and Southern Africa (3). In China, 477,900 patients were diagnosed with esophageal cancer and $\sim 375,00$ patients succumbed to the disease in 2015 (4). The prognosis of ESCC is very poor, with a 5-year survival rate following surgery, chemotherapy or radiotherapy treatment of only $15-25 \%$. As metastases are often present prior to diagnosis (5), it is crucial to understand the molecular mechanisms of metastasis in ESCC.

Tumor metastasis is the movement of tumor cells from a primary site to colonize distant organs progressively (6). The process of tumor metastasis is complicated and is associated with tumor microenvironment (7), changes in the cytoskeleton (8), activation or inactivation of signaling pathways (9), angiogenesis (10), epithelial-mesenchymal transition (EMT) process (11), cell proliferation, as well as the mobilization of oncogenes and the restrain of tumor suppressors (12). In the course of tumor progression, epithelial cells can exhibit plasticity and transition into a mesenchymal state by activating the EMT process, while cells in the mesenchymal state have a greater ability to migrate and invade (11). The Wnt/ $\beta$-catenin signaling pathway plays an important role in the process of cell proliferation and differentiation, while abnormal activation of this pathway can lead to tumor (13). A previous study reported that EMT could be regulated by Wnt/ $\beta$-catenin signal pathway (14), and revealed a new mechanism of tumor metastasis.

DJ-1 is an oncogene that is highly expressed in numerous cancer types compared with corresponding normal tissue, such 
as in lung cancer, breast cancer and endometrial cancer (15-17). The effects of DJ-1 in cancer are possibly through its ability to transform normal cells, prevent oxidative damage, and inhibit apoptosis and promote metastasis (18). DJ-1 has been reported to be an independent prognostic indicator of poor survival in ESCC (19). However, the mechanisms of DJ-1 that promote ESCC metastasis remain unclear. The present study aimed to investigate the role of DJ-1 in ESCC tumor cell proliferation and invasion, and its underlying mechanisms.

\section{Materials and methods}

Patient information and tissue specimens. The samples for the present study were collected from 84 patients diagnosed with ESCC who underwent surgical treatment at the Department of Thoracic Surgery, The Affiliated Hospital of Yangzhou University (Yangzhou, China) between January 2015 to December 2016. The age range was 49-81 years (median, 64.46 years), and 58 male and 26 females were included. None of the patients received radiotherapy or chemotherapy prior to surgery. The ESCC specimens obtained from thoracoscopic surgery were stored at $-80^{\circ} \mathrm{C}$, fixed in $10 \%$ formalin at room temperature for $24 \mathrm{~h}$ and embedded in paraffin. The study was reviewed and approved by the Institutional Ethics Committee of the Affiliated Hospital of Yangzhou University (Yangzhou, China). All patients signed informed consent forms.

Reagents and antibodies. UltraVision Quanto Detection system HRP DAB was obtained from Thermo Fisher Scientific, Inc. Roswell Park Memorial Institute-1640 (RPMI-1640) and fetal bovine serum (FBS) were purchased from Gibco; Thermo Fisher Scientific, Inc. Recombinant transforming growth factor- $\beta 1$ (TGF- $\beta 1$ ) was acquired from R\&D Systems. Matrigel was obtained from BD Biosciences. Anti-DJ-1 antibody was obtained from Santa Cruz Biotechnology, Inc (catalog no. sc-55572; 1:1,000 for western blotting; 1:200 for immunohistochemistry). Anti-E-Cadherin antibody was acquired from Abcam (catalog no. ab231303; 1:1,000 for western blotting; 1:1,000 for immunohistochemistry). Antibodies against vimentin (catalog no. 5741; 1:1,000 for western blotting; 1:200 for immunohistochemistry), N-Cadherin (catalog no. 13116S; 1:1,000 for western blotting), $\beta$-actin (catalog no. 4970S; 1:1,000 for western blotting), low density lipoprotein receptor-related protein 6 (LRP6) (catalog no. 2560S; 1:1,000 for western blotting), phosphorylation-LRP6 (p-LRP6) (catalog no. 2568S; 1:1,000 for western blotting), axin1 (catalog no. 2087S; 1:1,000 for western blotting) and $\beta$-catenin (catalog no. $8480 \mathrm{~S} ; 1: 1,000$ for western blotting; 1:100 for immunohistochemistry) were purchased from Cell Signaling Technology, Inc. HRP-conjugated goat anti mouse IgG (catalog no. G1006-1; 1:2,000 for western blotting) and HRP-conjugated goat anti-rabbit IgG (catalog no. HA1001; 1:2,000 for western blotting) were from Hangzhou Hua-an Medical \& Health Instruments Co., Ltd. Other chemicals of analytical grade were obtained from commercial sources.

Immunohistochemistry. Immunohistochemical staining of human paraffin-embedded ESCC was performed using
UltraVision Quanto Detection system HRP DAB, according to the manufacturer's protocol. Briefly, slides were cut to a $5-\mu \mathrm{m}$ thickness. Following deparaffinization and rehydration with gradient alcohol ranging between 100 and $70 \%$, the tissues were incubated in digestive enzyme for pretreatment. Sections were washed with PBS at room temperature, and then incubated in Hydrogen Peroxide Block for $10 \mathrm{~min}$ and Ultra $\mathrm{V}$ Block for $5 \mathrm{~min}$ at $37^{\circ} \mathrm{C}$ to reduce non-specific background staining. Following washing with PBS, the sections were incubated with primary antibodies against E-cadherin, vimentin, DJ-1 and b-catenin for $20 \mathrm{~min}$ at $37^{\circ} \mathrm{C}$. Following washing, the sections were then incubated with Applied HRP Polymer Quanto for $10 \mathrm{~min}$ at $37^{\circ} \mathrm{C}$. DAB Quanto Chromogen (30 $\mu \mathrm{l}$ ) was added to $1 \mathrm{ml}$ DAB Quanto Substrate, mixed by swirling and applied to tissue for $5 \mathrm{~min}$, followed by washing with distilled water at room temperature. Finally, the sections were counterstained with hematoxylin for $1 \mathrm{~min}$ at room temperature followed by dehydration and coverslip mounting. Negative controls were treated with PBS in place of primary antibodies. The stained slides were observed with a light microscope (magnification, x100 and x200) and evaluated using Image Pro Plus (version 6.0; Media Cybernetics) as previously described (20).

Cell lines and cell culture. The human ESCC cell line ECA-109 was purchased from the Type Culture Collection of the Chinese Academy of Sciences and cultured in RPMI-1640 medium containing $10 \% \mathrm{FBS}$ and $1 \%$ penicillin-streptomycin mixture. Cells were maintained at $37^{\circ} \mathrm{C}$ in a humidified atmosphere containing 5\% $\mathrm{CO}_{2}$. Prior to experiments, ECA-109 cells in the log phase of growth were cultured in six-well plates in media containing only $1 \%$ FBS for $24 \mathrm{~h}$.

EMT model. ECA-109 cells $\left(1 \times 10^{5}\right)$ were plated in six-well plates $12 \mathrm{~h}$ before the experiment, and RPMI-1640 containing $10 \mu \mathrm{g} / 1 \mathrm{TGF}-\beta 1$ was subsequently added to each well for $24 \mathrm{~h}$ under normal culture conditions (21). The cell morphology and distribution were captured under a light microscope (magnification, x200; Olympus Corporation). Subsequently, the protein expression levels of EMT-associated proteins E-cadherin, N-cadherin, vimentin and DJ-1 were detected by western blotting.

Lentivirus-mediated DJ-1 overexpression and knockdown. A lentiviral vector carrying green fluorescent protein (GFP) for DJ-1 overexpression (LV-DJ-1) was applied to mediate DJ-1 overexpression and an empty lentiviral vector was applied as the negative control (LV-con). A lentiviral vector carrying GFP and encoding short interfering RNA (siRNA) targeting DJ-1 was applied to mediate DJ-1 knockdown (LV-siRNA-DJ-1) and an empty lentiviral vector was used as the negative control (LV-siRNA-con). All lentivirus vectors were constructed by Shanghai Genechem Co., Ltd. GV358 lentiviral vector was used to upregulate the expression of DJ-1 and GV248 lentiviral vector was used to knockdown the expression of DJ-1. The negative controls were also generated using GV248 and GV358, respectively. The primer sequence of DJ-1 was as follow: Forward, 5'-GAGGATCCCCGGGTACCGGTCGCCACCATG GCTTCCAAAAGAGCTCTGG-3' and reverse, 5'-TCCTTG TAGTCCATACCGTCTTTAAGAACAAGTGGAG-3'. The 
SiRNA sequence was 5'-TTAGAGAAACAGGCCGTTA-3'. ECA-109 cells $\left(1.0 \times 10^{6}\right)$ were seeded in six-well plates, and lentivirus (MOI, 10) with viral-plus transduction enhancer and polybrene (Shanghai Genechem Co., Ltd.) at a final concentration of $8 \mu \mathrm{g} / \mathrm{ml}$ were added and incubated at $37^{\circ} \mathrm{C}$ for $48 \mathrm{~h}$. Subsequently, the cells were treated with $10 \mathrm{~g} / \mathrm{ml}$ puromycin to eliminate the uninfected cells for $72 \mathrm{~h}$. The GFP positive cells were evaluated using a fluorescence microscope (Olympus Corporation) and transfection was further confirmed by western blotting.

Western blot analyses. Total proteins of each cell group were prepared using RIPA buffer containing $1 \mathrm{mmol} / \mathrm{l}$ PMSF and a protease inhibitor cocktail (Beyotime Institute of Biotechnology). Proteins were quantified using the BCA assay kit (Beyotime Institute of Biotechnology). Total proteins $(30 \mu \mathrm{g} /$ lane $)$ from each lysate were separated by $10 \%$ SDS-PAGE and then transferred onto PVDF membranes. Membranes were blocked in blocking buffer (5\% non-fat dry milk and 1\% Tween-20 in PBS) for $2 \mathrm{~h}$ at room temperature. The membranes were incubated with the primary antibodies against DJ-1, E-cadherin, vimentin, $\mathrm{N}$-cadherin, $\beta$-actin, LRP6, p-LRP6, Axin1 and $\beta$-catenin overnight at $4^{\circ} \mathrm{C}$, and then incubated with HRP-conjugated rabbit or anti-mouse antibody at room temperature for $2 \mathrm{~h}$. Immunoreactive signals were visualized using ECL detection reagent (Thermo Fisher Scientific, Inc.) with the Molecular Imager Chemi Doc XRS system (Bio-Rad Laboratories, Inc.). The bands were quantified using Quantity One analysis software version 4.62 (Bio-Rad Laboratories, Inc.). The value of each protein was normalized to the $\beta$-actin level in the same sample.

Cell proliferation assay. Thymidine analog 5-ethynyl-2'-deoxyuridine (EdU) (Invitrogen; Thermo Fisher Scientific, Inc.) was used to detect cell proliferation. According to the manufacturer's protocol, $2.0 \times 10^{4}$ cells/well were seeded into 96-well plates and incubated at $37^{\circ} \mathrm{C}$ in a $5 \% \mathrm{CO}_{2}$ incubator for $24 \mathrm{~h}$. Then, $25 \mu \mathrm{M}$ EdU was added for $2 \mathrm{~h}$ at $37^{\circ} \mathrm{C}$. Following washing with PBS, cells were fixed with $4 \%$ paraformaldehyde for $30 \mathrm{~min}$ at room temperature and terminated with $2 \mathrm{mg} / \mathrm{ml}$ glycine. The Apollo staining reaction liquid (catalog no. C10310; Guangzhou RiboBio Co., Ltd.) was then added to the wells and incubated in dark at room temperature for $30 \mathrm{~min}$. Subsequently, $100 \mu 10.5 \%$ TritonX-100 was applied to reduce the dye background. DAPI was diluted and used to dye the nucleus in dark at room temperature for $30 \mathrm{~min}$. The results were observed and photographed using a fluorescence inversion microscope system (magnification, x100; Olympus Corporation).

Cell clone assay. To examine clonogenic ability, 500 cells were seeded in six-well plates and cultured with complete medium for 2 weeks in a $5 \% \mathrm{CO}_{2}$ incubator at $37^{\circ} \mathrm{C}(22)$. Then, the cells were fixed with $99 \%$ methanol for $10 \mathrm{~min}$ at room temperature, and stained with $0.1 \%$ crystal violet (Sigma-Aldrich; Merck KGaA) for $15 \mathrm{~min}$ at room temperature. Clones with $\geq 50$ cells were scored under a light microscope (Olympus Corporation) and five random views were evaluated (magnification, x100).
Cell adhesion assay. The 96-well plates were coated with $0.04 \mu \mathrm{g} / \mu \mathrm{l}$ Matrigel (BD Biosciences) prior to the experiments. Cells $\left(5 \times 10^{4}\right)$ were seeded in the plates for $2 \mathrm{~h}$ and then washed with PBS to remove non-adherent cells. MTT $(5 \mathrm{mg} / \mathrm{ml}$; Sigma-Aldrich; Merck KGaA) was added to each well in the dark for $4 \mathrm{~h}$ in a $5 \% \mathrm{CO}_{2}$ incubator at $37^{\circ} \mathrm{C}$. The optical density (OD) value was measured at $490 \mathrm{~nm}$. The adhesion rate was calculated as: OD of objective cells/OD of negative control cells $\mathrm{x} 100 \%$.

Transwell assay. A Corning Matrigel invasion chamber (Corning, Inc.) was used for the Transwell assay. In the invasion assay, Matrigel gel diluted with serum-free RPMI-1640 (1:8) was added to the upper chamber. A single cell suspension in serum-free RPMI-1640 was added to the upper chamber at a density of $2 \times 10^{4}$ cells/well and incubated for $24 \mathrm{~h} \mathrm{(23).}$ The non-invading cells on the upper side of the chamber were removed using cotton swabs. The membrane containing the invaded cells was fixed with $99 \%$ methanol for $10 \mathrm{~min}$ at room temperature and stained with $0.1 \%$ crystal violet for $10 \mathrm{~min}$ at room temperature. Migration assays were performed using the same procedure, except that the polycarbonate membrane was not coated with Matrigel. Each experiment was repeated three times. The number of invading and migrating cells was detected by light microscopy (magnification, x200). The invasion and migration capacity of cells was calculated as follows: Cell number of the treatment group/cell number of the control group $\mathrm{x} 100 \%$.

Inhibitor treatment. To evaluate the exact mechanism, the present study investigated the effect of $\beta$-catenin inhibition on the role of DJ-1 in ESCC malignant behaviors using XAV939 (MedChemExpress), a small molecule inhibitor of Wnt/ $\beta$-catenin. LV-DJ-1 cells were cultured in 6-well plates in advance, then $10 \mathrm{mM}$ XAV939 was added for $24 \mathrm{~h}$ in a $5 \% \mathrm{CO}_{2}$ incubator at $37^{\circ} \mathrm{C}$ and the same concentration of DMSO was used as a negative control. The treated cells were then subjected to a cell proliferation assay, cell clone assay, cell adhesion assay, Transwell assay and western blotting.

Nude mice intraperitoneal xenograft model. A total of 20 4-week-old female athymic nude BALB/c mice were obtained from the Comparative Medicine Laboratory Animal Center of Yangzhou University (Jiangsu, China). All mice were raised in a specific pathogen-free atmosphere environment at $25^{\circ} \mathrm{C}$ with a $12 \mathrm{~h}$ light/dark cycle and allowed free access to food and water. All animal experiments were approved by the Institutional Animal Care and Use Committee of Yangzhou University and performed in accordance with internationally accepted guidelines on the use of laboratory animals. Nude mice were randomly assigned to four groups; each group contained 5 mice. ECA-109/LV-con, ECA-109/LV-DJ-1, ECA-109/LV-siRNA-DJ-1 and ECA-109/LV-siRNA-con cells $\left(1 \times 10^{6}\right)$ were separately inoculated into the peritoneal cavity of nude mice (24). Because the intraperitoneal nodules could not be measured directly for tumor progression, we referred to relevant literature and chose 28 days as the total time of the experiment (24). In the 28 days, tumor 
A
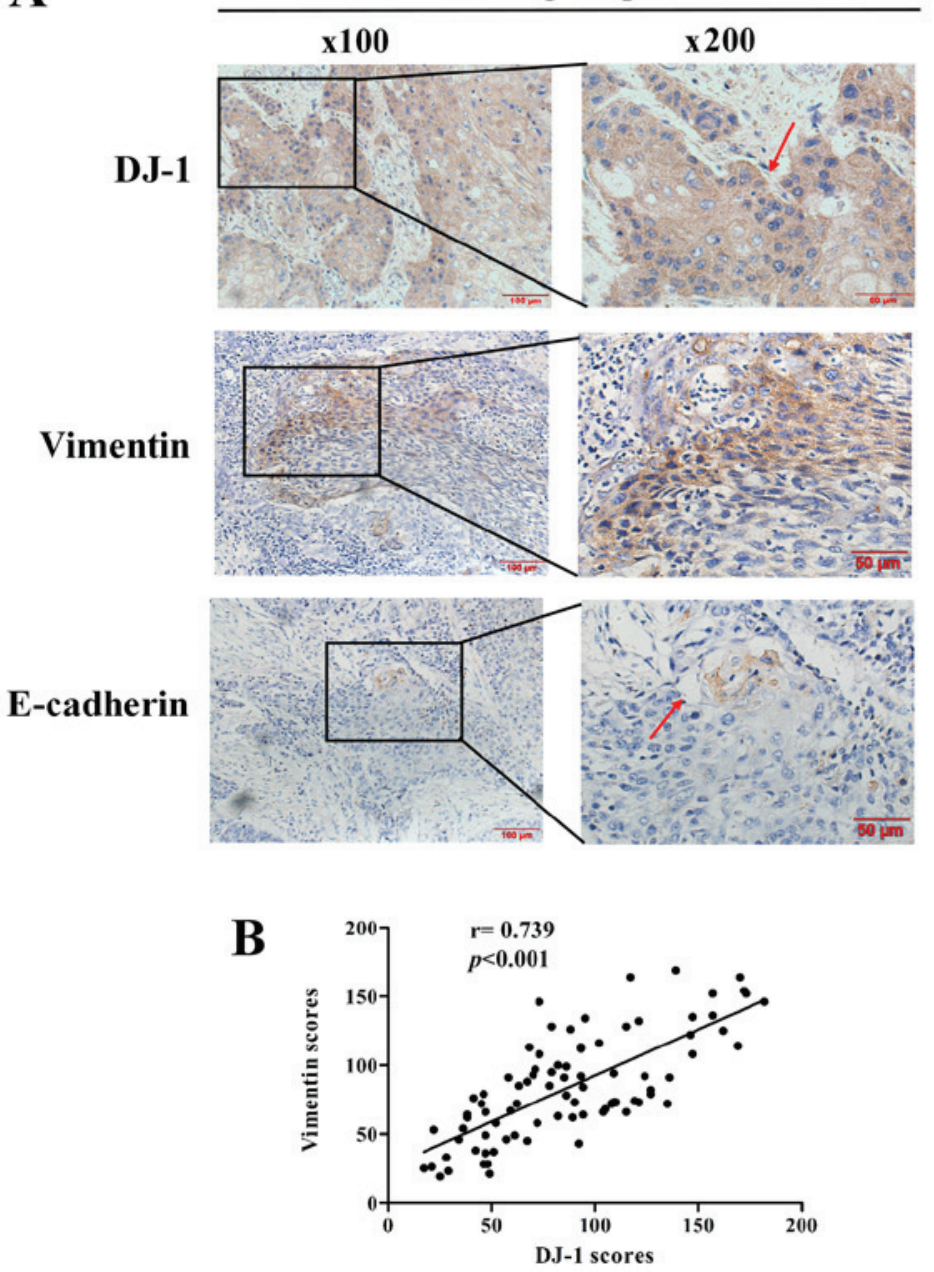

DJ-1 low expression
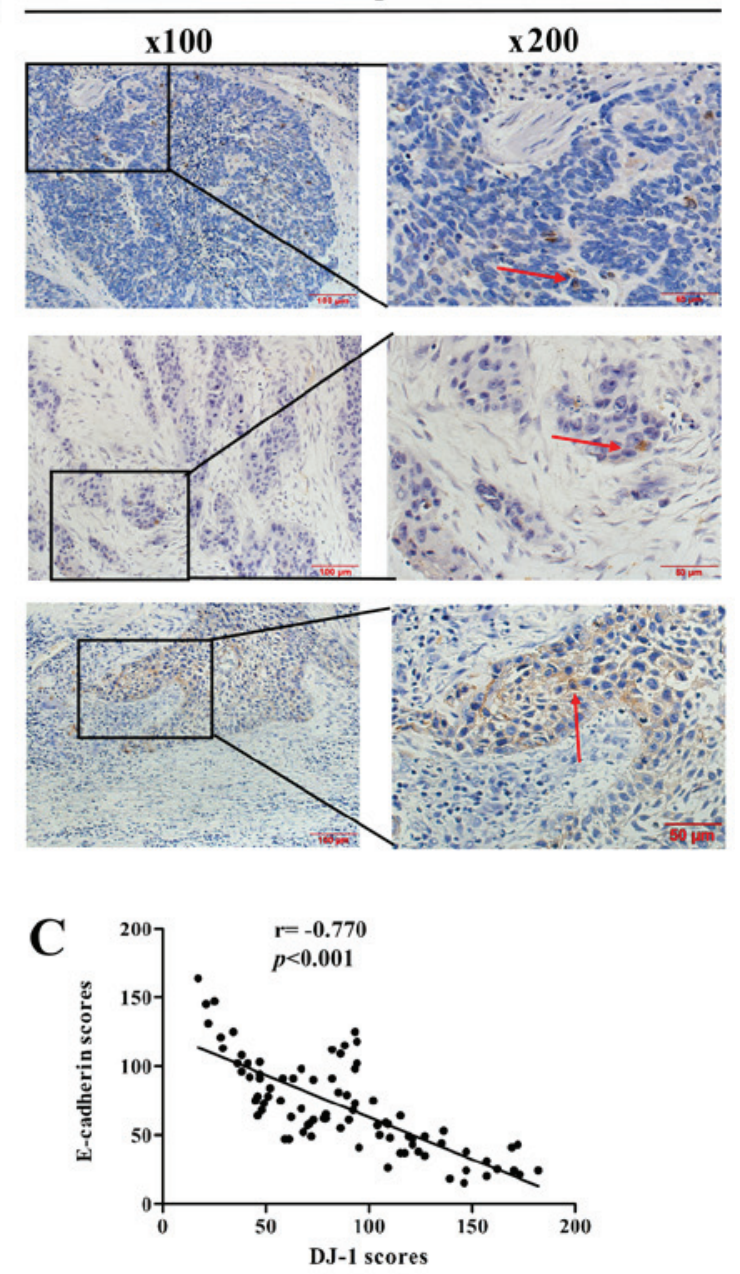

Figure 1. Immunohistochemical staining of DJ-1, vimentin and E-cadherin in ESCC specimens and the correlation between DJ-1 and EMT proteins. (A) Immunohistochemical staining of DJ-1, vimentin and E-cadherin in ESCC. The red arrows indicate positively stained cells. Scale bars, 100 or $50 \mu \mathrm{m}$. (B) DJ-1 was positively correlated with vimentin $(r=0.739, \mathrm{P}<0.001)$. (C) DJ-1 was negatively correlated with E-cadherin $(r=-0.770, P<0.001)$.

progression was assessed by abdominal palpation and in vivo imaging of the small animals using IVIS $^{\circledR}$ Lumina Series III (PerkinElmer, Inc.) every 2 days. Isoflurane (3\%) was used for induction and $1.5 \%$ isoflurane was used for maintenance of anesthesia in the process of imaging small animals in vivo. However, due to the poor imaging effect of GFP in cells and the high background fluorescence in the picture, tumor progress was roughly evaluated according to the intensity of the strongest fluorescence. During the process, all the mice were alive, and almost all of them showed wasting, but they were able to eat and drink normally; therefore, the experiment was continued until the end of the experiment period. A total of 28 days following injection, all the mice were anesthetized with $3 \%$ isoflurane gas inhalation and sacrificed by cervical dislocation, then a laparotomy was performed. Peritoneal dissemination and organ metastasis were examined. The peritoneal nodules and liver were fixed in $10 \%$ formalin and embedded in paraffin for pathological analysis.

Statistical analyses. All the experiments were repeated three times. Protein expression levels and clinicopathological features were compared using the $\chi^{2}$ test. Correlations between
DJ-1, E-cadherin and vimentin in clinical samples were analyzed using Pearson's correlation analysis. Other data are presented as the mean \pm standard deviation, and groups were compared using Student's t-test. One-way ANOVA followed by Fisher's least significant difference post hoc test was used for multiple comparisons. All statistical analysis was performed using SPSS version 16.0 software (SPSS, Inc.). P $<0.05$ was considered to indicate a statistically significant difference.

\section{Results}

DJ-1 is associated with metastasis and EMT in clinical human esophageal squamous cancer patients. In order to elucidate the association between DJ-1 and EMT in ESCC, immunohistochemistry analyses were first performed to detect the expression levels of DJ-1, vimentin and E-cadherin in 84 human ESCC tissue specimens (Fig. 1A). The associations between the clinical pathological features of patients with ESCC and the DJ-1, E-cadherin ad vimentin immunohistochemical staining scores are summarized in Table I. The data demonstrated that high expression of DJ-1 was significantly associated with the lymph node metastasis and distant metastasis. In addition, high expression of vimentin and low 
Table I. Associations between DJ-1, E-cadherin and vimentin expression and the clinicopathological features of 84 patients with esophageal squamous cell carcinoma.

\begin{tabular}{|c|c|c|c|c|c|c|c|c|c|c|c|c|c|}
\hline \multirow[b]{2}{*}{ Parameter } & \multirow[b]{2}{*}{$\mathrm{n}$} & \multicolumn{4}{|c|}{ DJ-1 expression } & \multicolumn{4}{|c|}{ Vimentin expression } & \multicolumn{4}{|c|}{ E-cadherin expression } \\
\hline & & High & Low & $\chi^{2}$ & P-value & High & Low & $\chi^{2}$ & P-value & High & Low & $\chi^{2}$ & P-value \\
\hline Age, years & & & & 0.350 & 0.554 & & & 0.239 & 0.625 & & & 0.075 & 0.784 \\
\hline$\leq 60$ & 15 & 7 & 8 & & & 8 & 7 & & & 6 & 9 & & \\
\hline$>60$ & 69 & 38 & 31 & & & 32 & 37 & & & 25 & 44 & & \\
\hline Sex & & & & 0.257 & 0.612 & & & 0.032 & 0.857 & & & 0.472 & 0.492 \\
\hline Male & 58 & 30 & 28 & & & 28 & 30 & & & 20 & 38 & & \\
\hline Female & 26 & 15 & 11 & & & 12 & 14 & & & 11 & 15 & & \\
\hline Tumor size, $\mathrm{cm}$ & & & & 1.440 & 0.230 & & & 1.925 & 0.165 & & & 0.614 & 0.433 \\
\hline$\leq 5$ & 48 & 23 & 25 & & & 26 & 22 & & & 16 & 32 & & \\
\hline$>5$ & 36 & 22 & 14 & & & 14 & 22 & & & 15 & 21 & & \\
\hline Location & & & & 3.127 & 0.209 & & & 1.359 & 0.507 & & & 0.470 & 0.791 \\
\hline Upper & 14 & 8 & 6 & & & 7 & 7 & & & 6 & 8 & & \\
\hline Middle & 39 & 17 & 22 & & & 16 & 23 & & & 13 & 26 & & \\
\hline Lower & 31 & 20 & 11 & & & 17 & 14 & & & 12 & 19 & & \\
\hline Differentiation & & & & 4.503 & 0.105 & & & 7.489 & 0.024 & & & 8.531 & 0.014 \\
\hline Well & 7 & 8 & & & & 5 & 10 & & & 10 & 5 & & \\
\hline Moderate & 25 & 27 & & & & 22 & 30 & & & 18 & 34 & & \\
\hline Poor & 13 & 4 & & & & 13 & 4 & & & 3 & 14 & & \\
\hline pT status & & & & 3.489 & 0.062 & & & 4.587 & 0.032 & & & 4.741 & 0.029 \\
\hline $\mathrm{T} 1-2$ & 24 & 9 & 15 & & & 7 & 17 & & & 13 & 11 & & \\
\hline $\mathrm{T} 3-\mathrm{T} 4$ & 60 & 36 & 24 & & & 33 & 27 & & & 18 & 42 & & \\
\hline Lymph node metastasis & & & & 6.489 & 0.011 & & & 7.126 & 0.008 & & & 6.347 & 0.012 \\
\hline No & 29 & 10 & 19 & & & 8 & 21 & & & 16 & 13 & & \\
\hline Yes & 55 & 35 & 20 & & & 32 & 23 & & & 15 & 40 & & \\
\hline Metastasis & & & & 8.526 & 0.004 & & & 9.185 & 0.002 & & & 10.181 & 0.001 \\
\hline No & 46 & 18 & 28 & & & 15 & 31 & & & 24 & 22 & & \\
\hline Yes & 38 & 27 & 11 & & & 25 & 13 & & & 7 & 29 & & \\
\hline
\end{tabular}

expression of E-cadherin were significantly associated with the tumor cell differentiation, pT status, lymph node metastasis and distant metastasis $(\mathrm{P}<0.05)$.

Furthermore, the expression levels of EMT biomarkers E-cadherin and vimentin were significantly correlated with DJ-1. As presented in Fig. 1B and C, DJ-1 expression was positively correlated with vimentin expression $(r=0.739, \mathrm{P}<0.001)$ and negatively correlated with E-cadherin $(\mathrm{r}=-0.770, \mathrm{P}<0.001)$. These results collectively suggest that DJ-1 may be closely associated with metastasis and the EMT in ESCC.

DJ-1 is highly expressed during EMT in a ESCC cell model. To study the DJ-1 expression in EMT progress, EMT was induced in ECA-109 cells using $10 \mathrm{ng} / \mathrm{ml}$ TGF- $\beta 1$. Following $24 \mathrm{~h}$, cells were photographed under a light microscope and examined by western blotting. As presented in Fig. 2A, the cell morphology of the TGF- $\beta 1$ group exhibited spindle-like shapes. The distribution of cells in the TGF- $\beta 1$ group was more extensive and changes in migration patterns were observed compared with the control group. Furthermore, the expression levels of EMT biomarkers were significantly different following
TGF- $\beta 1$ treatment; E-cadherin was significantly decreased, and $\mathrm{N}$-cadherin and vimentin were significantly increased $(\mathrm{P}<0.01)$. In addition, DJ-1 expression was significantly increased following TGF- $\beta 1$ treatment $(\mathrm{P}<0.01$; Fig. $2 \mathrm{~B})$. Thus, DJ-1 was highly expressed during EMT in ECA-109 cells.

DJ-1 overexpression promotes cell growth of ESCC cells. To validate the role of DJ-1 in tumor metastasis and EMT, lentivirus vectors were used to overexpress DJ-1 in Eca-109 cells. Successful overexpression of DJ-1 of was verified by fluorescence observation of the GFP signal (Fig. 3A). In addition, western blotting demonstrated that DJ-1 expression was significantly increased in LV-DJ-1 cells compared with control cells $(\mathrm{P}<0.01$; Fig. 3B). As presented in Fig. 3C, cell proliferation was higher following DJ-1 overexpression compared with the control group, as the Edu staining was stronger. In order to further observe the change of cell proliferation ability, a cell clone assay was performed. This revealed that LV-DJ-1 cells had a larger colony size and significantly more colonies $(189.33 \pm 15.50)$ compared with the control cells $(106 \pm 12.77)$ $(\mathrm{P}<0.01$; Fig. 3D). 
A

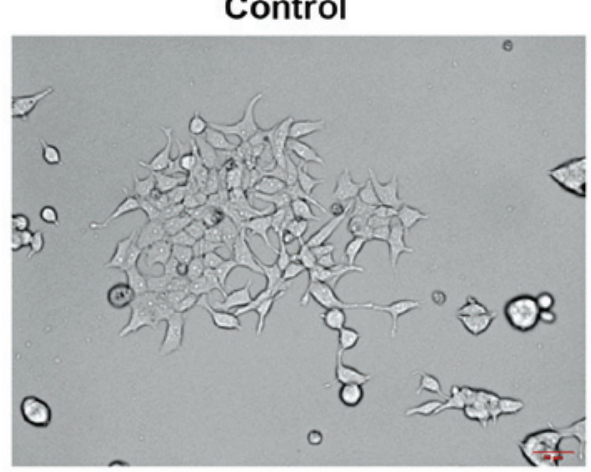

TGF- $\beta 110 \mathrm{ng} / \mathrm{ml}$

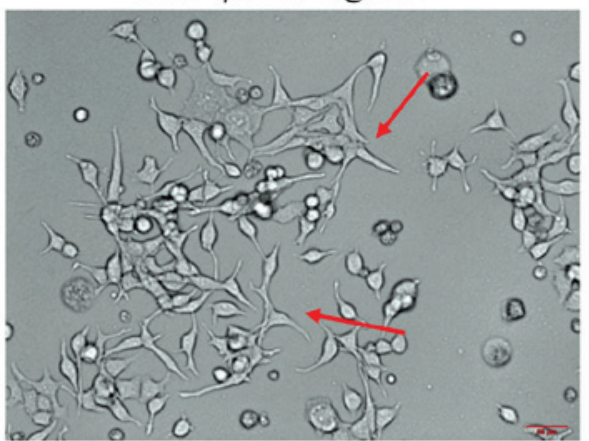

\section{B Control TGF- $\beta 110 \mathrm{ng} / \mathrm{ml}$}

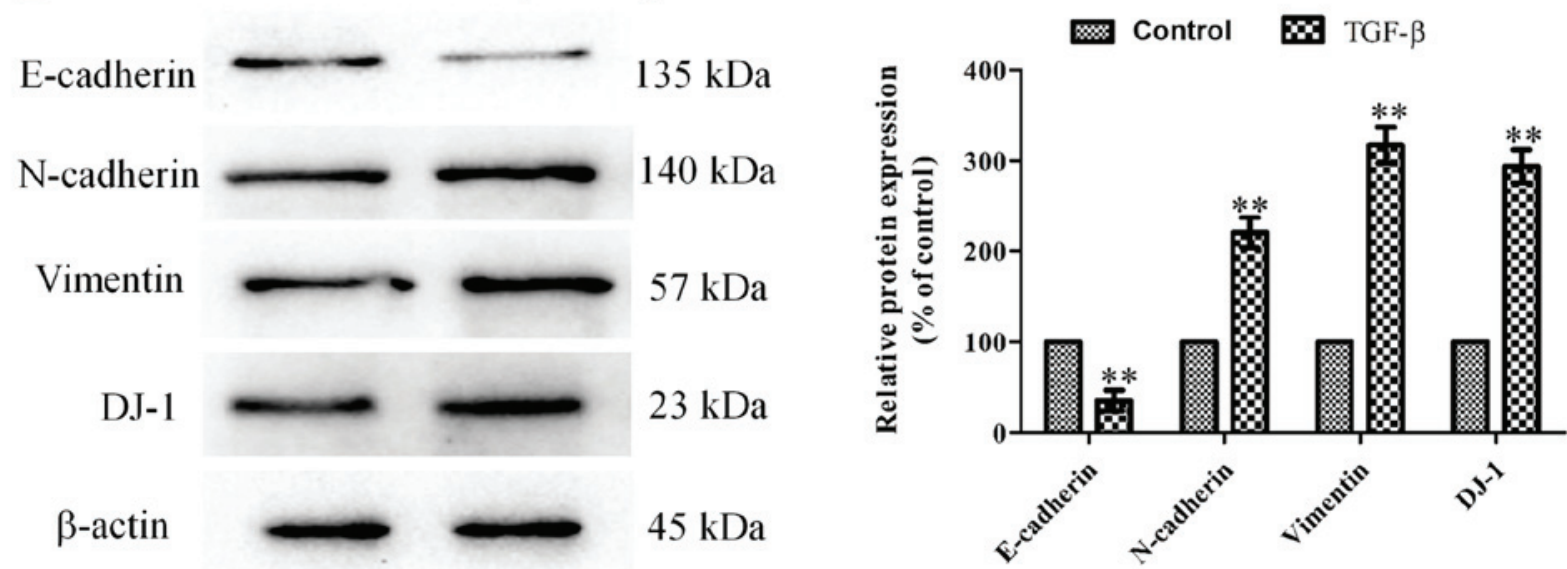

Figure 2. DJ-1 is highly expressed in the EMT model. (A) The morphology of cells in each group (magnification, x200). (B) Expression levels of EMT proteins and DJ-1 in each group. E-cadherin expression was significantly decreased in TGF- $\beta 1$-treated cells, while N-cadherin, vimentin and DJ-1 expression levels were significantly increased. ${ }^{* *} \mathrm{P}<0.01$ vs. control. TGF- $\beta 1$, transforming growth factor- $\beta 1$.
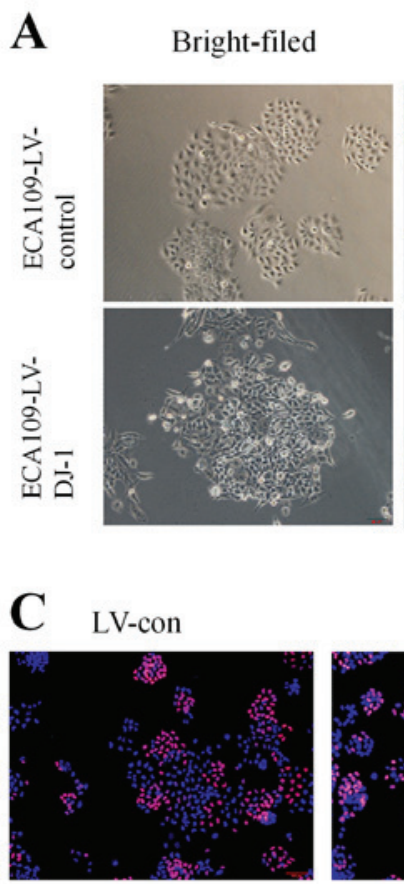

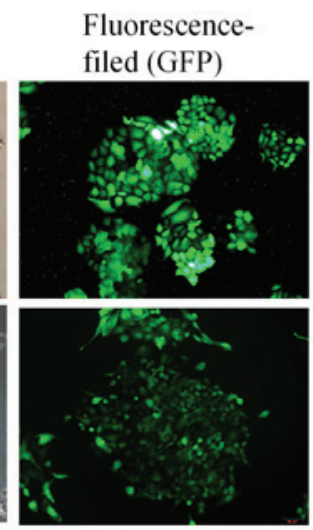

Merge layers

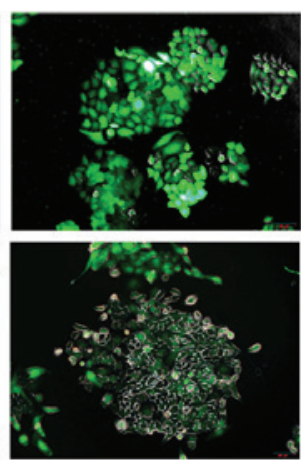

D

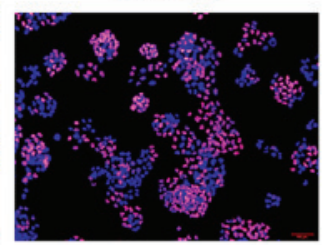

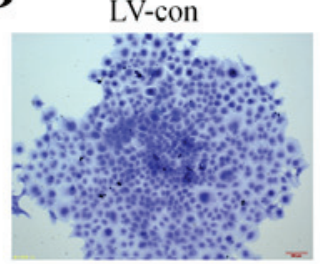

B
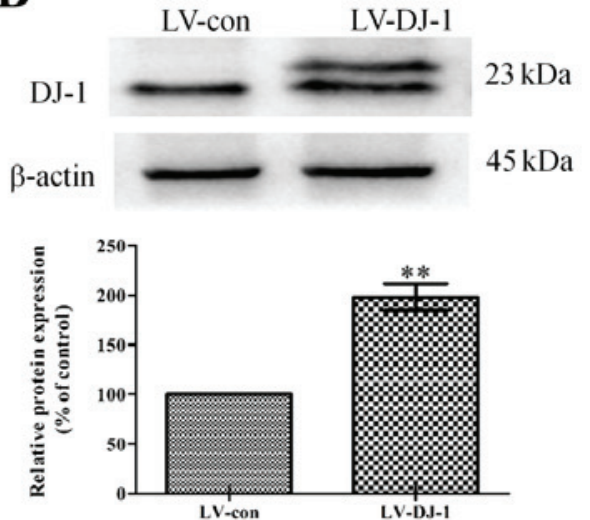

LV-DJ-1

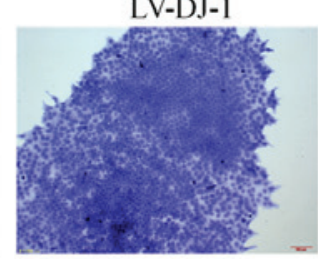

Figure 3. Establishment of the DJ-1-overexpression cell model, and analysis of the proliferation and colony formation in LV-DJ-1 and control cells. (A) The morphological changes of ECA-109 cells after transfection with LV-DJ-1. Scale bars, $50 \mu \mathrm{m}$ (magnification, x200). (B) DJ-1 expression was significantly higher in LV-DJ-1 cells compared with control cells. (C) DJ-1 overexpression promoted ESCC cell proliferation (magnification, 100X). (D) DJ-1 overexpression promoted colony formation of ESCC cells (magnification, $\mathrm{x} 100$ ). ${ }^{* *} \mathrm{P}<0.01 \mathrm{vs}$. LV-con. LV-DJ-1, lentivirus overexpressing DJ-1; LV-con, lentivirus control. 
A

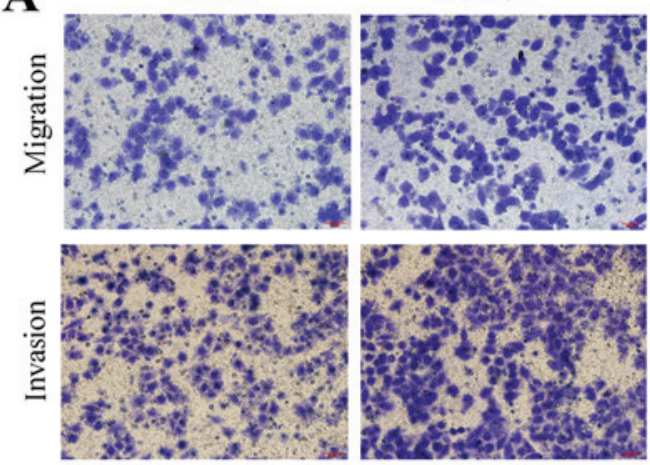

C

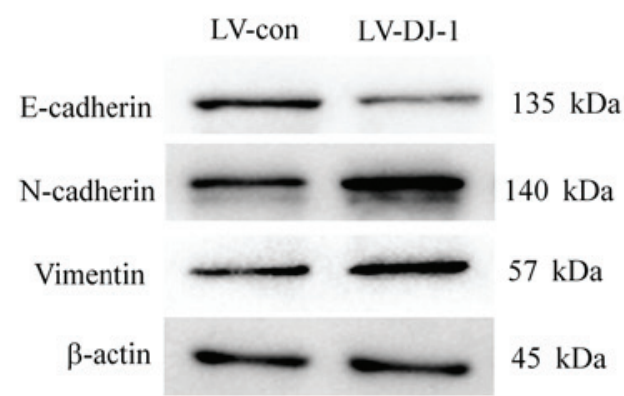

D

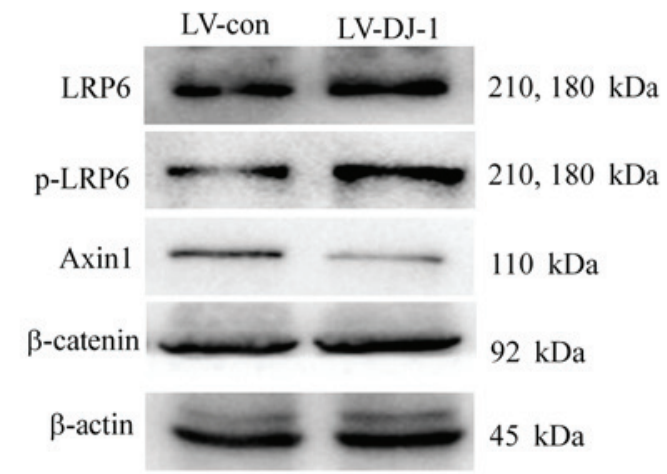

B
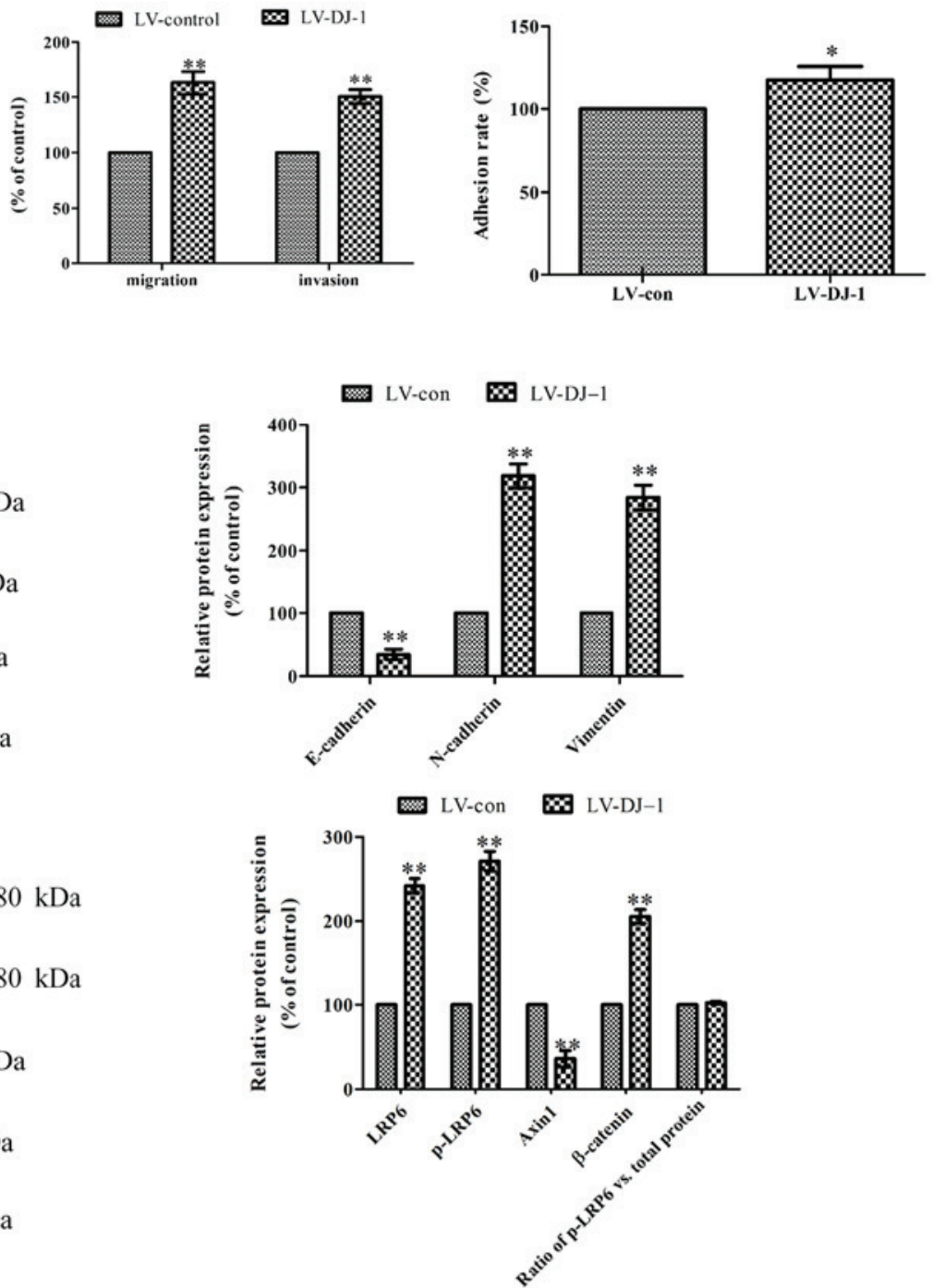

Figure 4. DJ-1 promotes EMT via the Wnt/ $\beta$-catenin signaling pathway in esophageal squamous cell carcinoma. (A) LV-DJ-1 cells had a significantly higher migration and invasion ability compared with the control group (magnification, x200). (B) The adhesion ability was significantly increased in LV-DJ-1 cells. (C) DJ-1 overexpression promoted the EMT process by significantly reducing E-cadherin expression and significantly increasing the vimentin and N-cadherin expression. (D) LRP6, p-LRP6 and $\beta$-catenin expression were significantly upregulated, while Axin1 expression was significantly downregulated in LV-DJ-1 cells. The ratio of p-LRP6 to total LRP6 protein exhibited no significant change between the control group and $\mathrm{DJ}-1$ overexpression group. ${ }^{*} \mathrm{P}<0.05,{ }^{* *} \mathrm{P}<0.01$ vs. LV-con. LV-DJ-1, lentivirus overexpressing DJ-1; LV-con, lentivirus control; LRP-6, lipoprotein receptor-related protein 6; p-. phosphorylated.

DJ-1 overexpression promotes migration, invasion, adhesion and EMT via the Wnt/b-catenin signaling pathway of ESCC cells. A Transwell assay was performed to detect the migration and invasion ability of DJ-1 overexpressed cells. As presented in Fig. 4A, LV-DJ-1 cells had a significantly higher migration and invasion ability compared with the LV-con group $(\mathrm{P}<0.01)$. Similarly, the adhesion rate was significantly higher in LV-DJ-1 cells compared with the LV-con cells $(\mathrm{P}<0.05$; Fig. 4B). Western blotting results demonstrated that DJ-1 overexpression could significantly reduce the E-cadherin expression, and significantly increase the vimentin and $\mathrm{N}$-cadherin expression $(\mathrm{P}<0.01$; Fig. $4 \mathrm{C})$, which indicates DJ-1 promotes the EMT process. LRP6, p-LRP6, axin1 and $\beta$-catenin are key proteins of the canonical Wnt/ $\beta$-catenin signal pathway (13). It was identified that in LV-DJ-1 cells, LRP6, p-LRP6 and $\beta$-catenin expression levels were significantly upregulated, while axin1 expression was significantly downregulated compared with control cells (P<0.01; Fig. 4D). However, the ratio of p-LRP6 to total LRP6 protein had no significant change between the control group and DJ-1 overexpressed group ( $\mathrm{P}>0.05$; Fig. 4D). Therefore, this suggests that a high expression of DJ-1 does not effect the phosphorylation of LPR6.

DJ-1 knockdown suppresses cell growth of ESCC cells. To further confirm the effects of DJ-1 in tumor metastasis and EMT of ESCC, lentivirus vectors encoding siRNAs targeting DJ-1 were used to knockdown DJ-1 expression in ECA-109 cells. As presented in Fig. 5A, DJ-1 was knocked-down, according to the extent of green fluorescence. Additionally, western blot assay revealed that the protein expression of DJ-1 was significantly reduced in LV-siRNA-con cells $(\mathrm{P}<0.01$; 


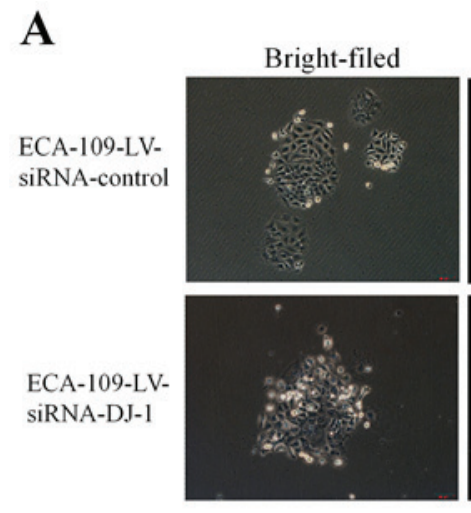

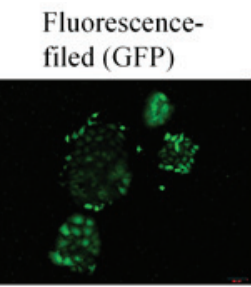

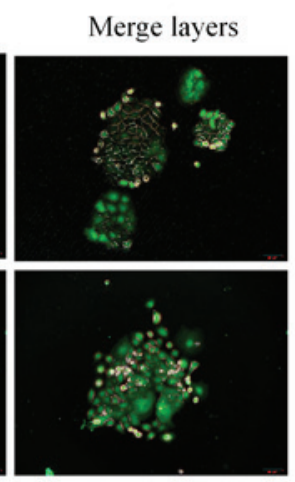

$\mathbf{B}$
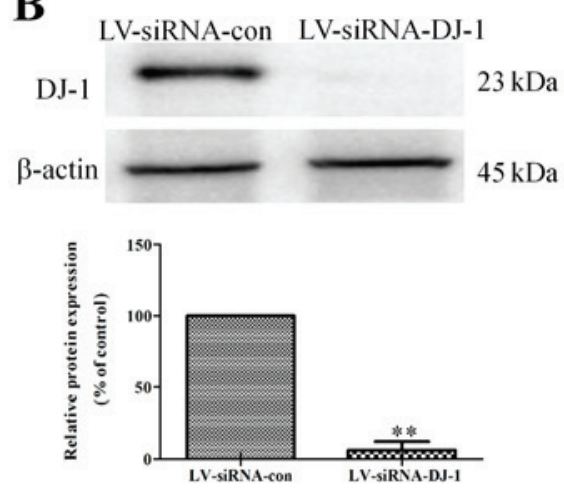
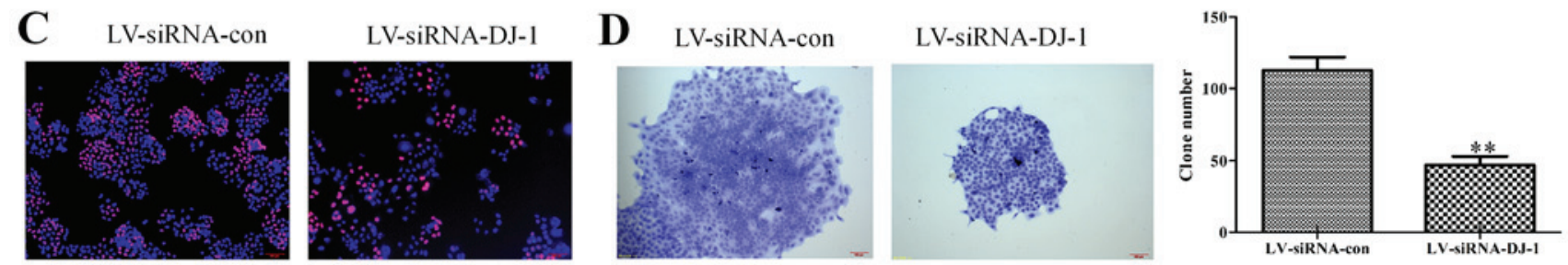

Figure 5. DJ-1 knockdown restrains cell proliferation and colony formation. (A) The morphological changes of ECA-109 cells after transfection with LV-siRNA-DJ-1. Scale bar, $100 \mu \mathrm{m}$ (magnification, x100). (B) DJ-1 expression was significantly lower in LV-siRNA-DJ-1 cells compared with the control. (C) DJ-1 knockdown inhibited ESCC cell proliferation (magnification, x100). (D) DJ-1 knockdown inhibited colony formation of ESCC cells (magnification, $\mathrm{x} 100) .{ }^{* *} \mathrm{P}<0.01$ vs. LV-siRNA-con. LV-siRNA-DJ-1, lentivirus encoding DJ-1 small interfering RNA; LV-siRNA-con, lentivirus encoding control small interfering RNA; GFP, green fluorescent protein; ESCC, esophageal squamous cell carcinoma.

Fig. 5B). These results suggest that the DJ-1 low-expression cell model was successfully constructed. Edu staining assay demonstrated that LV-siRNA-DJ-1 cells had a low proliferative capacity compared with control cells, as the red fluorescence was enhanced. (Fig. 5C). Furthermore, the ability of cells in the LV-siRNA-DJ-1 group to proliferate and form clones was significantly lower compared with the control group $(\mathrm{P}<0.01$; Fig. 5D).

DJ-1 knockdown suppresses migration, invasion, adhesion and EMT via the Wnt/ $\beta$-catenin signaling pathway in ESCC cells. In contrast to DJ-1 overexpressed cells, the LV-siRNA-DJ-1 cells had significantly lower migration, invasion and adhesion abilities compared with LV-siRNA-control cells $(\mathrm{P}<0.01$; Fig. 6A and $\mathrm{B})$. The E-cadherin expression was significantly higher, while $\mathrm{N}$-cadherin and Vimentin expression were significantly lower in LV-siRNA-DJ-1 cells compared with control cells $(\mathrm{P}<0.01$; Fig. 6C). Furthermore, LRP6, p-LRP6 and b-catenin expression were significantly downregulated, while Axin 1 expression was significantly upregulated in LV-siRNA-DJ-1 cells ( $\mathrm{P}<0.01$; Fig. 6D). Finally, the ratio of phosphorylated LRP6 to total LRP6 protein was significantly increased in LV-siRNA-DJ-1 cells compared with control cells.

Inhibition of Wnt/ $\beta$-catenin reduces tumor malignant behaviors induced by $D J-1$. In order to investigate the exact mechanism of the Wnt/ $\beta$-catenin signaling pathway in the regulation of DJ-1 in tumor malignant behaviors, the $\beta$-catenin inhibitor XAV939 was used in in vitro studies. LV-DJ-1 cells were treated with XAV939; as presented in Fig. 7A, the proliferation of LV-DJ-1 cells treated with XAV939 was decreased compared with LV-DJ-1 cells. In the colony formation assay, cells treated with XAV939 had a significantly smaller number of colonies compared with the control LV-DJ-1 cells $(\mathrm{P}<0.01$; Fig. 7B). In the Transwell assay, LV-DJ-1 cells treated with XAV939 demonstrated significantly reduced abilities to migrate and invade compared with LV-con group ( $\mathrm{P}<0.01$; Fig. 7C). Similarly, the adhesion ability was significantly decreased in the XAV939 group compared with the LV-con group ( $\mathrm{P}<0.01$; Fig. 7D). Western blotting results demonstrated that XAV939 treatment could significantly increase the E-cadherin, while it significantly reduced the vimentin and $\mathrm{N}$-cadherin expression levels compared with the untreated LV-DJ-1 cells $(\mathrm{P}<0.01$; Fig. 7E), which indicates XAV939 inhibited the EMT process promoted by DJ-1. Additionally, LRP6, p-LRP6 and b-catenin expression levels were significantly downregulated, while Axin 1 expression was significantly upregulated compared with control cells, and the ratio of phosphorylated LRP6 to total LRP6 protein was significantly increased in XAV939-treated cells compared with the control untreated LV-DJ-1 cells $(\mathrm{P}<0.05$; Fig. 7F), These data suggest XAV939 could reverse tumor malignant behavior induced by overexpression of DJ-1.

Manipulation on DJ-1 expression influences ESCC xenograft metastasis and EMT via the Wnt/ $\beta$-catenin signaling pathway. Since the important roles of DJ-1 in metastasis and EMT have been demonstrated by clinical analysis and in vitro experiments, its biological effect on ESCC metastasis and EMT was further examined in vivo using a nude mice abdominal transplantation model. In vivo imaging of the small animals was also used to observe tumor growth dynamically. However, due to the poor imaging effect of green fluorescent protein in cells and the high background fluorescence in the picture, tumor progress was roughly estimated according to 
A

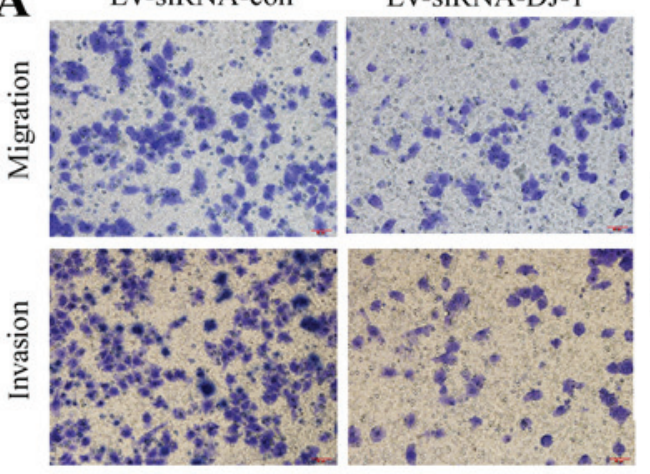

C

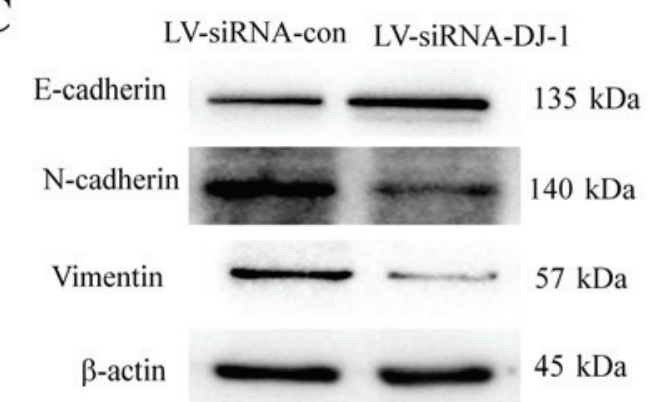

D

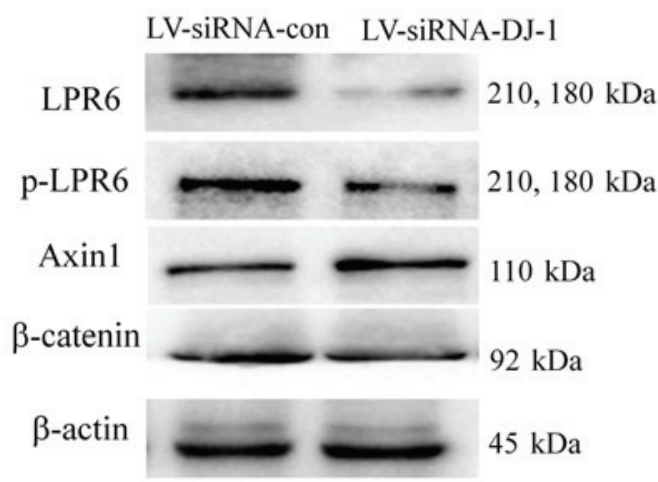

B
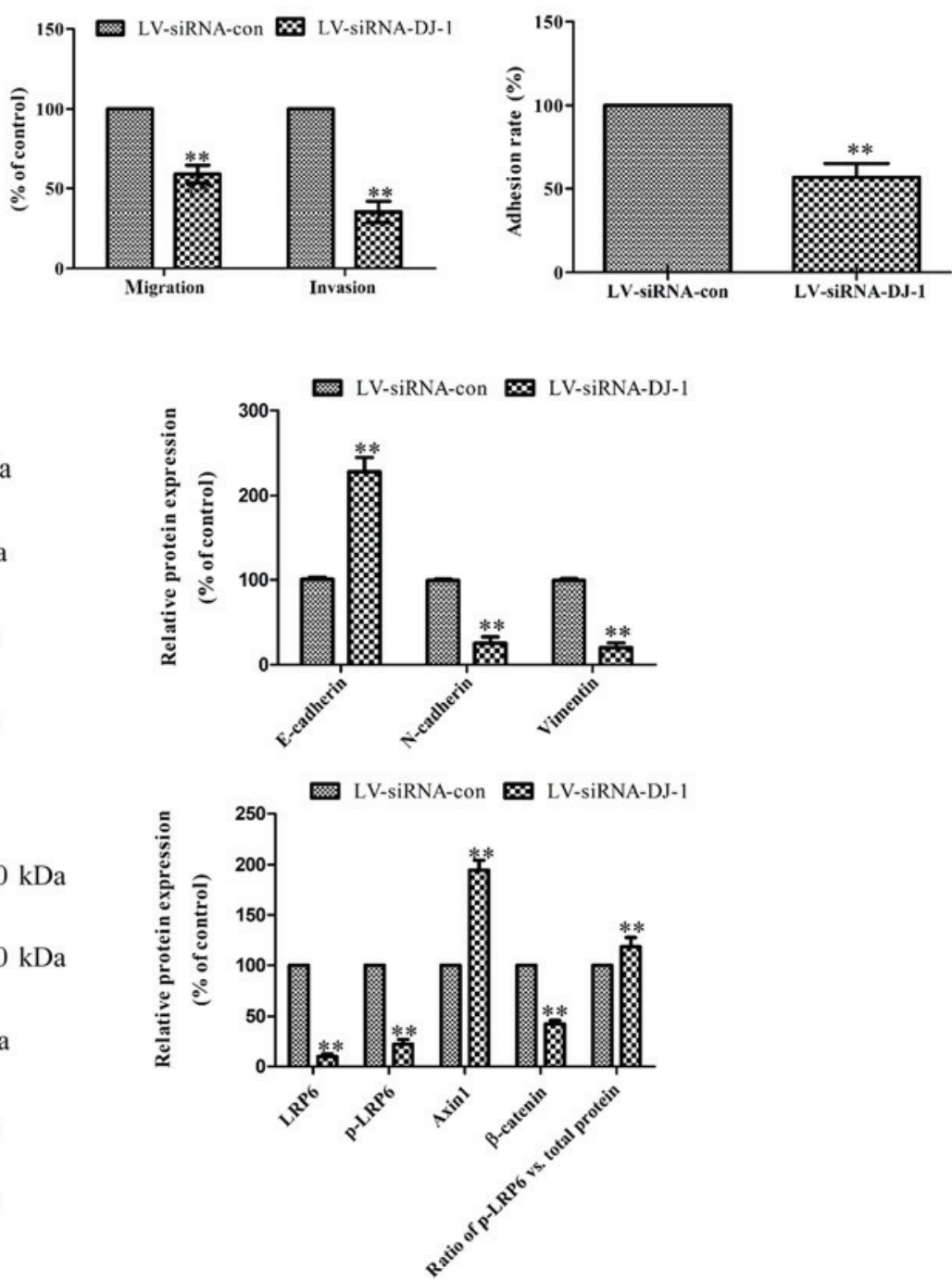

Figure 6. DJ-1 knockdown suppresses the EMT process via the Wnt/3-catenin signaling pathway in ESCC. (A) LV-siRNA-DJ-1 cells had a lower migration and invasion ability compared with the control group (magnification, x200). (B) Adhesion ability was significantly decreased in LV-siRNA-DJ-1 cells. (C) DJ-1 knockdown suppressed the EMT process in ESCC cells by increasing the E-cadherin expression and reducing the vimentin and N-cadherin expression. (D) LRP6, p-LRP6 and $\beta$-catenin expression were downregulated, while Axin1 expression was upregulated in LV-siRNA-DJ-1 cells compared with control cells. The ratio of p-LRP6 to total LRP6 protein was significantly increased in LV-siRNA-DJ-1 cells compared with control cells. ${ }^{* *} \mathrm{P}<0.01 \mathrm{vs.} \mathrm{LV-siRNA-con}$. ESCC, esophageal squamous cell carcinoma; LV-siRNA-DJ-1, lentivirus encoding DJ-1 small interfering RNA; LV-siRNA-con, lentivirus encoding control small interfering RNA; LRP-6, lipoprotein receptor-related protein 6; p-. phosphorylated.

the intensity of the strongest fluorescence. In the late stage of tumor growth, differences were observed between each group. At day 28, the LV-DJ-1 group had a larger area and stronger fluorescence compared with the control, while the LV-siRNA-DJ-1 group had a smaller area of fluorescence (Fig S1). The numbers of peritoneal dissemination nodules were examined and the liver metastases were removed and analyzed. As presented in Fig. 8A and B, the number of peritoneal dissemination nodules in LV-DJ-1 group was significantly increased compared with the LV-con group, while in the LV-siRNA-DJ-1 group, the number of peritoneal dissemination nodules was significantly reduced compared with the LV-siRNA-con group $(\mathrm{P}<0.01)$. Notably, liver metastases in the LV-DJ-1 group exhibited a larger volume and contained a larger number of nodules (Fig. 8C). By contrast, in the
LV-siRNA-DJ-1 group, liver metastases had a smaller volume and fewer nodules (Fig. 8D). Immunohistochemistry results further explained the mechanism of DJ-1 promoting tumor metastasis. Immunohistochemical staining (Fig. 8E) revealed increased DJ-1, vimentin and $\beta$-catenin levels, and decreased E-cadherin levels in the LV-DJ-1 group compared with the control group. In addition, staining demonstrated decreased DJ-1, vimentin and $\beta$-catenin levels, and increased E-cadherin levels in the LV-siRNA-DJ-1 group compared with the control group.

\section{Discussion}

The role of DJ-1 has been extensively investigated in numerous human cancer types. In the process of tumor progression, DJ-1 

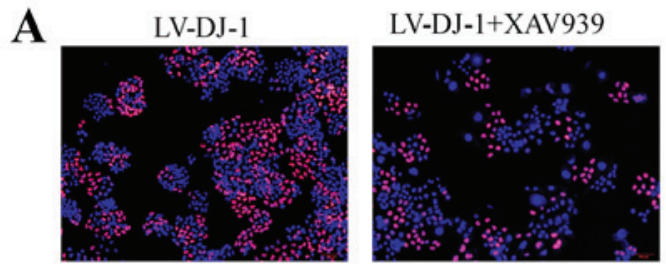

C

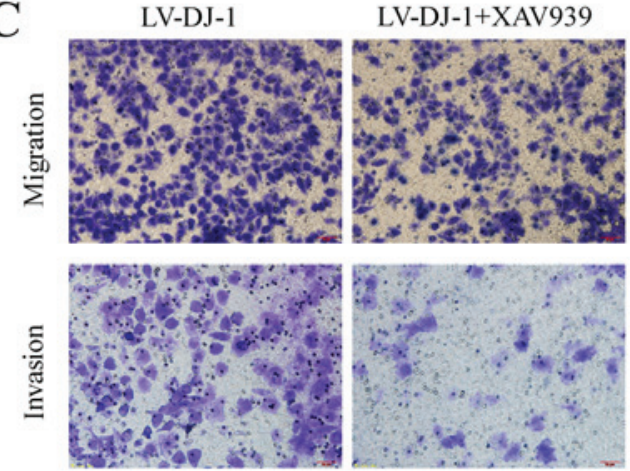

$\mathbf{E}$

LV-DJ-1 LV-DJ-1+XAV939

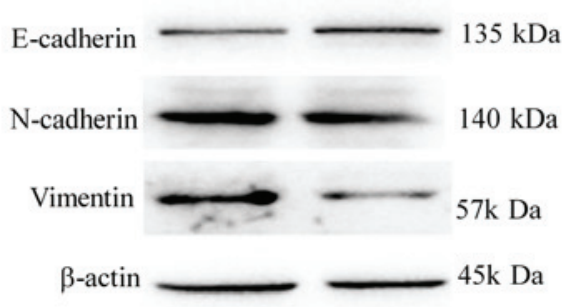

$\mathbf{F}$
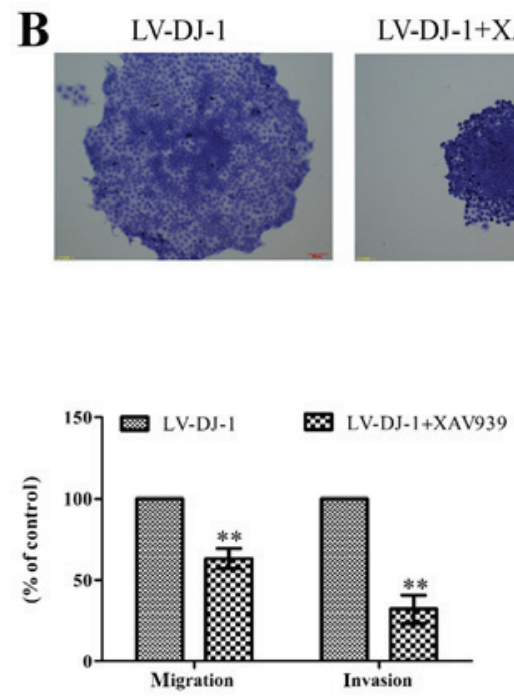

LV-DJ-1+XAV939

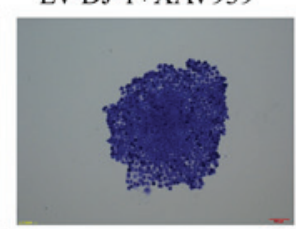

D

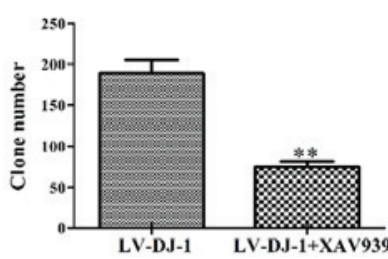

LV-DJ-1 LV-DJ-1+XAV93
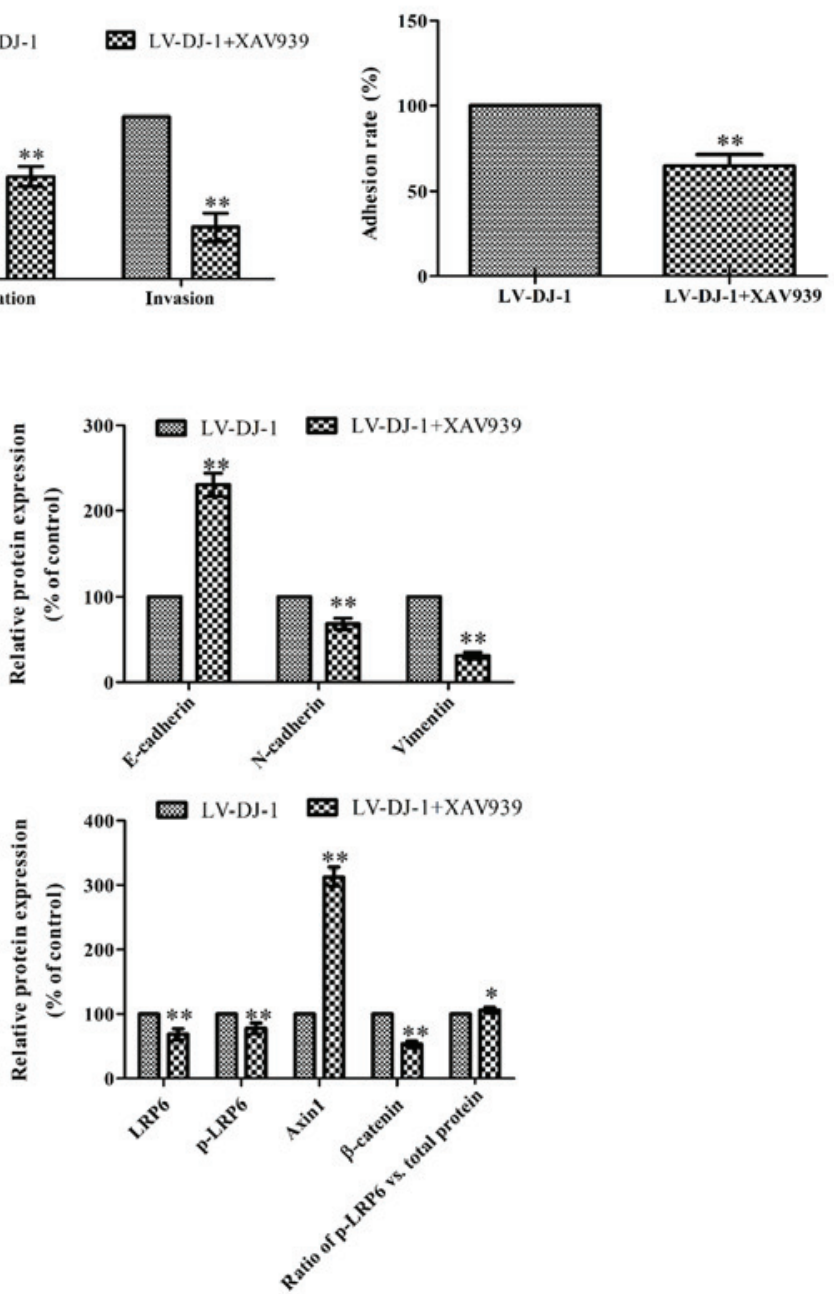

Figure 7. Inhibition of Wnt/B-catenin reduces tumor malignant behaviors caused by DJ-1. (A) The proliferation of LV-DJ-1 cells treated with XAV939 was significantly decreased compared with LV-DJ-1 cells (magnification, x100). (B) In the colony formation assay, cells treated with XAV939 had fewer colonies compared with the control LV-DJ-1 cells (magnification, x100). (C) In the Transwell assay, cells of the LV-DJ-1 + XAV939 group demonstrated a weaker ability to migrate and invade compared with the LV-DJ-1 group (magnification, x200). (D) The adhesion ability was decreased in the LV-DJ-1 + XAV939 group compared with the LV-DJ-1 group. (E) Western blotting results demonstrated XAV939 treatment could increase the E-cadherin expression, while it reduced the vimentin and N-cadherin expression levels. (F) LRP6, p-LRP6 and $\beta$-catenin expression were downregulated, while Axin1 expression was upregulated in XAV939-treated compared with control cells. The ratio of p-LRP6 to total LRP6 protein was significantly increased in XAV939-treated cells compared with the control untreated LV-DJ-1 cells. ${ }^{*} \mathrm{P}<0.05,{ }^{* *} \mathrm{P}<0.01$ vs. LV-DJ-1 group. LV-DJ-1, lentivirus overexpressing DJ-1; LRP-6, lipoprotein receptor-related protein 6; p-. phosphorylated.

activates the AKT/mTOR and MAPK signaling pathway by suppressing the PTEN gene, and promotes proliferation, survival, anti-apoptosis, invasion and metastasis of tumor cells $(25,26)$. In addition, DJ-1 serves as an endogenous antioxidant to protect cells from oxidative stress via oxidizing itself and/or stabilizing the antioxidant transcriptional responses regulator Nrf2 (27). A previous study reported that cytoplasmic DJ-1 expression is significantly higher in ESCC and ESCC lymph node metastases compared with in non-neoplastic esophageal epithelium. ESCC specimens with high distant metastatic potential also had a significantly higher level of nuclear DJ-1 expression (19). However, the mechanisms of DJ-1 promoting esophageal squamous carcinoma metastasis remain unclear.

The present study investigated the role of DJ-1 in regulation of the EMT process and metastasis, and clarified its potential mechanisms in ESCC. To understand the association between DJ-1 and EMT, the current study investigated the expression 
A

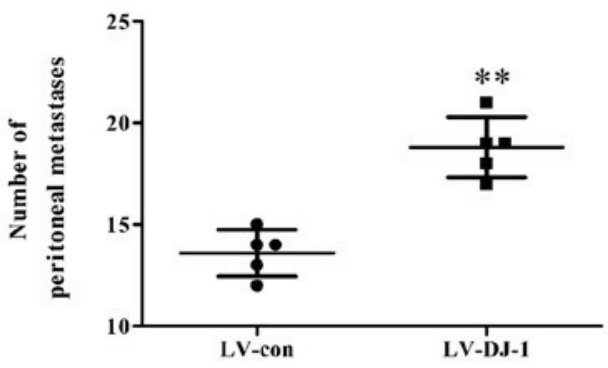

C

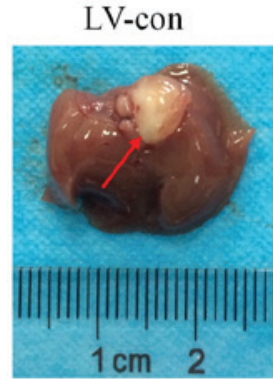

$\mathbf{E}$

DJ-1

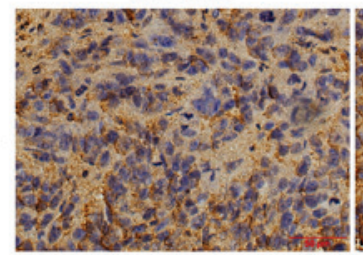

LV-con

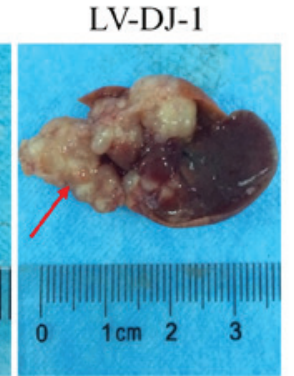

LV-DJ-1

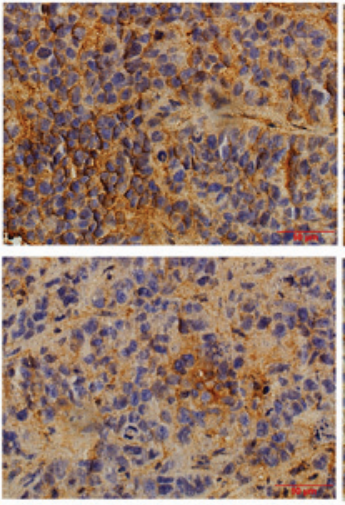

\section{E-cadherin}

$\beta$-catenin
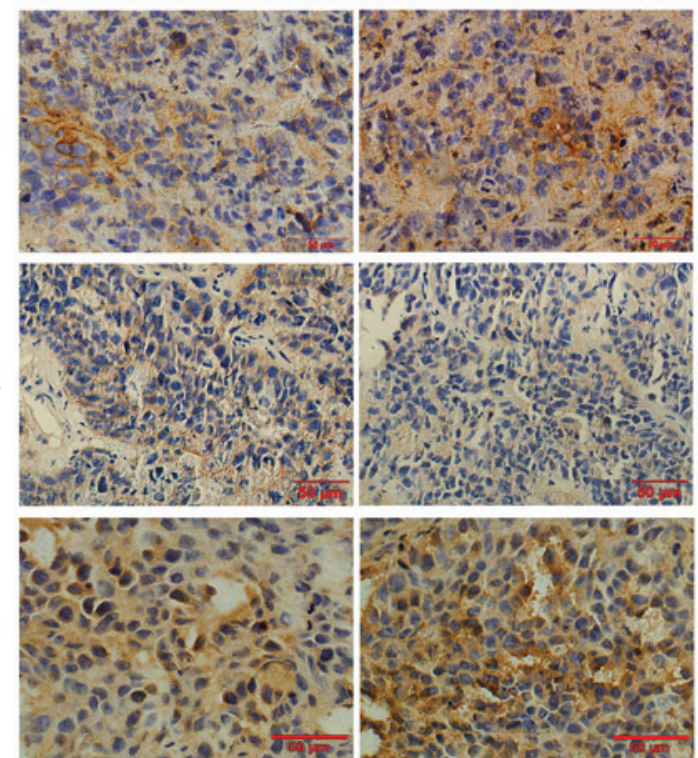

B

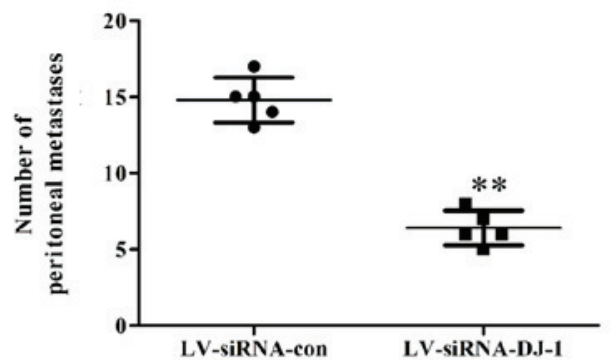

D

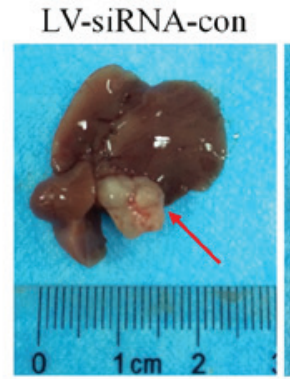

LV-siRNA-DJ-1

LV-siRNA-con
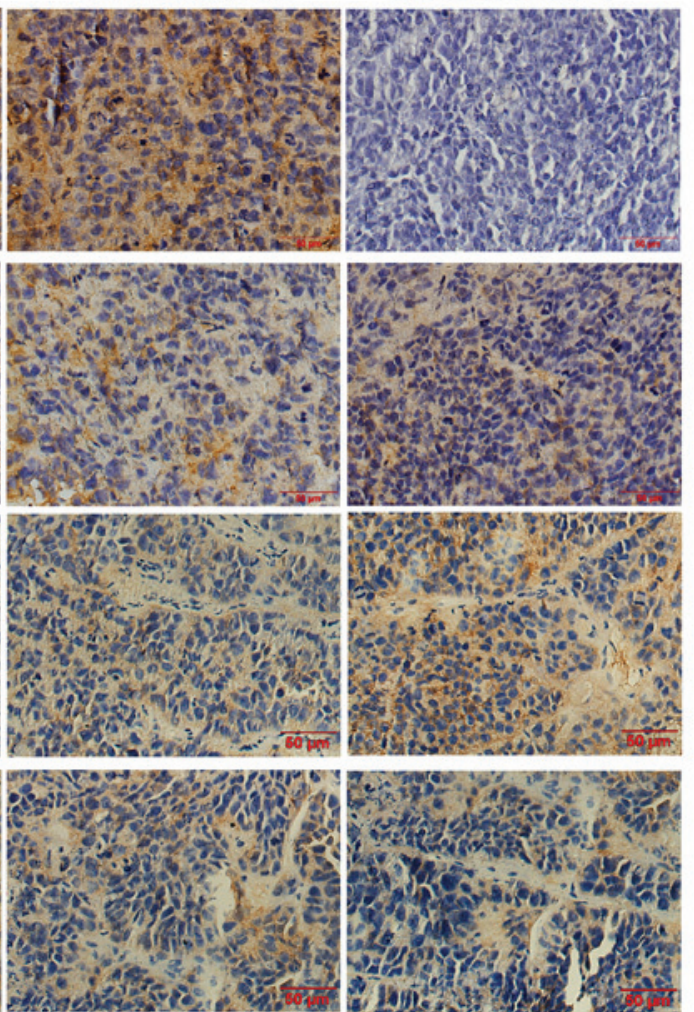

Figure 8. Manipulation of DJ-1 expression influences esophageal squamous cell carcinoma xenograft metastasis and EMT via the Wnt/ $\beta$-catenin signal pathway. (A) The number of peritoneal dissemination nodules in the LV-DJ-1 group was significantly higher compared with the LV-con group. ${ }^{* *} \mathrm{P}<0.01 \mathrm{vs}$. LV-con. (B) In LV-siRNA-DJ-1 group, the number of peritoneal dissemination nodules was significantly lower compared with the LV-siRNA-con group. ${ }^{* *} \mathrm{P}<0.01$ vs. LV-siRNA-DJ-1. (C) Liver metastases in the LV-DJ-1 group exhibited a larger volume and more nodules. (D) In the LV-siRNA-DJ-1 group, liver metastases exhibited a smaller volume and fewer nodules. (E) Immunohistochemistry results revealed increased DJ-1, vimentin and $\beta$-catenin, and decreased E-cadherin levels in the LV-DJ-1 group, and decreased DJ-1, vimentin and $\beta$-catenin, and increased E-cadherin levels in the LV-siRNA-DJ-1 group (magnification, x200). LV-DJ-1, lentivirus overexpressing DJ-1; LV-con, lentivirus control; LV-siRNA-DJ-1, lentivirus encoding DJ-1 small interfering RNA; LV-siRNA-con, lentivirus encoding control small interfering RNA.

levels of DJ-1, the mesenchymal cell marker vimentin and the epithelial cell marker E-cadherin in 84 paraffin-embedded ESCC tissue samples by immunohistochemistry. It was identified that DJ-1 was significantly associated with local lymph node metastasis and distant metastasis, while the vimentin and E-cadherin were significantly associated with tumor cell differentiation, depth of tumor invasion, lymph node metastasis and distant metastases. Furthermore, DJ-1 was positively correlated with vimentin and a negatively correlated with E-cadherin $(\mathrm{P}<0.01)$. Furthermore, DJ-1 was highly 
expressed in the TGF- $\beta 1$-induced EMT model of ECA-109 cells. Altogether, the present data demonstrated that DJ-1 was closely associated with tumor metastasis and EMT. To further investigate the effects of DJ-1 on metastasis and EMT, DJ-1 was upregulated and knocked-down in ECA-109 cells using lentivirus vectors. Cell proliferation is an important process for successful tumor transformation (28). Cells with DJ-1 overexpression had a greater proliferation rate and colony formation ability compared with the control cells, while DJ-1 knockdown resulted in a slower proliferation rate and colony formation compared with the control. Tumor cells gradually migrate to the vicinity of blood vessels and invade blood vessels, travel with the blood for adhesion, and then proliferate to form new metastases (29). The present results demonstrated that overexpression of DJ-1 could increase the adhesion, migration and invasion. By contrast, the adhesion, migration and invasion were significantly decreased in the DJ-1 low-expression group. Notably, DJ-1 promoted EMT by increasing $\mathrm{N}$-cadherin and vimentin expression levels, and reducing E-cadherin expression.

The Wnt/ $\beta$-catenin signaling pathway serves a fundamental role in the cell fate specification during early embryonic development, proliferation, body axis patterning, survival, apoptosis, and in tissue homeostasis in adults (30). A mutated Wnt pathway leads to multiple growth-related pathologies and cancer (31). Zhou et al (32) found that DJ-1 can promote CRC metastasis by activating the PLAGL2-Wnt-BMP4 axis (32). This result suggested that DJ-1 could regulate the $\mathrm{Wnt} / \beta$-catenin signaling pathway in cancer. However, it remains unclear whether DJ-1 can inhibit the invasion and metastasis in ESCC by regulating the $\mathrm{Wnt} / \beta$-catenin signaling pathway. Therefore, the present study further investigated the role and mechanism of DJ-1 and the Wnt/ $\beta$-catenin signaling pathway in the invasion and metastasis of ESCC. Canonical Wnt/ $/$-catenin signaling begins with secreted Wnt ligands binding to the Frizzled family receptors, and then the LRP5/LRP6 co-receptor triggers the $\beta$-catenin signaling cascade (33). Du et al (34) demonstrated that silencing LRP6 leads to less metastasis and angiogenesis in HCC cells. Tung et al (35) identified that regulation of LRP6 could promote hepato-carcinogenesis and enhance cell invasion. LRP6 phosphorylation is accompanied by receptor internalization in caveolin-containing vesicles and endocytosis, which is essential for Wnt/ $\beta$-catenin signaling (36). Tang et al (37) reported that $\beta$-catenin expression has higher in ESCC tissues compared with corresponding normal mucosa tissues, and exhibited a correlation with histological grade and invasion depth. Axin1 protein negatively regulates the Wnt signaling pathway based on its role as a scaffolding protein and its binding to cytoplasmic $\beta$-catenin (38).

The present study detected the expression levels of LRP6, p-LRP6, Axin1 and $\beta$-catenin in LV-DJ-1, LV-con, siRNA-DJ-1 and siRNA-con cells. The western blotting results demonstrated DJ-1 upregulation could increase LRP6, p-LRP6 and $\beta$-catenin levels, and decrease Axin1. Whereas, DJ-1-knockdown decreased the LRP6, p-LRP6 and $\beta$-catenin levels, and increased Axin1. These data revealed that the Wnt/ $\beta$-catenin signaling pathway participates in the process of DJ-1-induced regulation of tumor metastasis and EMT. These results were consistent with the data in literature that DJ-1 can promote CRC metastasis by activating the Wnt axis (32). The present study identified that the ratio of p-LRP6 to total LRP6 protein was not significantly different between the control group and DJ-1-overexpressing group. These results suggest that a high expression of DJ-1 did not result in changes in the phosphorylation of LPR6.

$\beta$-catenin protein is a core molecule and important regulatory site of the Wnt signaling pathway, and the level of $\beta$-catenin has a decisive influence on this pathway (39). It has been reported that $\beta$-catenin is one of the differentially expressed proteins between high-metastatic human ovarian cancer and non-metastatic ovarian cancer, and subsequent in vitro and in vivo studies have confirmed that overexpression of $\beta$-catenin can promote ovarian cancer metastasis, while knockdown of $\beta$-catenin expression can significantly weaken the cell metastasis ability (39). Therefore, the present study speculated that $\beta$-catenin plays an important role in the process of DJ-1 inhibiting Wnt/ $\beta$-catenin signal pathway. The $\beta$-catenin inhibitor XAV939 was used to further clarify the role of the Wnt/ $\beta$-catenin signaling pathway in DJ-1 promoting tumor metastasis. XAV939 is a novel small molecule inhibitor of the Wnt signaling pathway, which may restrain the abnormal activation of Wnt/ $\beta$-catenin and does not affect CRE, NF- $\kappa$ B or TGF- $\beta$ (40). Guo et al (41) found that XAV939 could suppress the viability of small cell lung cancer NCI-H446 cells and induce apoptosis. The present results demonstrated that XAV939 treatment could reduce the reverse tumor malignant behavior caused by overexpression of DJ-1. It has been reported that DJ-1-induced $\beta$-catenin nuclear translocation stimulates T-cell factor transcription activity, which promotes BMP4 expression for CRC cell migration and invasion, and elevates CCND1 expression for CRC cell proliferation (32). This report was consistent with the present results, which identified that DJ-1 inhibited ESCC cell invasion through the $\mathrm{Wnt} / \beta$-catenin pathway by inhibiting $\beta$-catenin. However, the current experiment focused on the relationship between DJ-1 and the invasion and metastasis of ESCC, and the mechanism of DJ-1 inhibiting $\beta$-catenin was not further investigated. The present results suggested that when the Wnt/ $\beta$-catenin signaling pathway was blocked, DJ-1 could not promote tumor metastasis as before. Furthermore, in vivo experiments were conducted using a nude mouse abdominal xenograft model. Data revealed that DJ-1 promoted a stronger metastasis ability and the EMT, and this result may be achieved by DJ-1 regulating the Wnt/ $\beta$-catenin signaling pathway.

In conclusion, the present study demonstrated that DJ-1 promoted tumor metastasis and EMT via the $\mathrm{Wnt} / \beta$-catenin signaling pathway in ESCC. These results indicate that DJ-1 may be a candidate therapeutic target for new ESCC drug development.

\section{Acknowledgements}

The authors would like to thank Dr Jun Feng (Gaoyou Traditional Chinese Medicine Hospital, Yangzhou, China) for providing assistance with the cell culture, and Mr. Yerong Yan (Comparative Medicine Laboratory Animal Center, Yangzhou University, Yangzhou, China) for taking care of the animals used in the study. 


\section{Funding}

This work was financially supported by the National Natural Science Foundation of China (grant nos. 81573656 and 81773944), the Natural Science Foundation of Jiangsu Province of China (grant no. BK20171290) and the Natural Science Foundation of Jiangsu Province for Youths (grant no. BK20170516).

\section{Authors' contributions}

FJ and HW designed the experimental procedures. FJ, DL, $\mathrm{CF}$ and WL jointly performed the experiments. QS, YD, ZD and FW helped in clinical specimen collection and performed statistical analysis. FJ, XD and MS analyzed the data. FJ, LT and YQ helped in designing the in vivo experiments and revised the manuscript critically for important intellectual content. YL contributed to the conception or design of the work and gave final approval of the version to be published. All authors reviewed the article and approved the final manuscript for publication.

\section{Availability of data and materials}

The datasets used and/or analyzed during the current study are available from the corresponding author on reasonable request.

\section{Ethics approval and consent to participate}

The animal experiments protocol was reviewed and approved by ethics committee of Medical College, Yangzhou University (Yangzhou, China). The human tissues study protocol was approved by the Institutional Ethics Committee of the Affiliated Hospital of Yangzhou University (Yangzhou, China), and informed consent was obtained from each patient.

\section{Patient consent for publication}

Not applicable.

\section{Competing interests}

The authors declare that they have no competing interests.

\section{References}

1. Lin DC, Wang MR and Koeffler HP: Genomic and epigenomic aberrations in esophageal squamous cell carcinoma and implications for patients. Gastroenterology 154: 374-389, 2018.

2. Torre LA, Bray F, Siegel RL, Ferlay J, Lortet-Tieulent J and Jemal A: Global cancer statistics, 2012. CA Cancer J Clin 65: 87-108, 2015.

3. Ferlay J, Soerjomataram I, Dikshit R, Eser S, Mathers C, Rebelo M, Parkin DM, Forman D and Bray F: Cancer incidence and mortality worldwide: Sources, methods and major patterns in GLOBOCAN 2012. Int J Cancer 136: E359-E386, 2015.

4. Chen W, Zheng R, Baade PD, Zhang S, Zeng H, Bray F, Jemal A, Yu XQ and He J: Cancer statistics in China, 2015. CA Cancer $\mathrm{J}$ Clin 66: 115-132, 2016.

5. Song Y, Li L, Ou Y, Gao Z, Li E, Li X, Zhang W, Wang J, Xu L, Zhou Y, et al: Identification of genomic alterations in oesophageal squamous cell cancer. Nature 509: 91-95, 2014.

6. Steeg PS: Targeting metastasis. Nat Rev Cancer 16: 201-218, 2016.
7. Joyce JA and Pollard JW: Micro-environmental regulation of metastasis. Nat Rev Cancer 9: 239-252, 2009.

8. Fife CM, McCarroll JA and Kavallaris M: Movers and shakers: Cell cytoskeleton in cancer metastasis. Br J Pharmacol 171: 5507-5523, 2014.

9. McCubrey JA, Abrams SL, Fitzgerald TL, Cocco L, Martelli AM, Montalto G, Cervello M, Scalisi A, Candido S, Libra M and Steelman LS: Roles of signaling pathways in drug resistance, cancer initiating cells and cancer progression and metastasis. Adv Biol Regul 57: 75-101, 2015.

10. Bonapace L, Coissieux MM, Wyckoff J, Mertz KD, Varga Z, Junt $\mathrm{T}$ and Bentires-Alj M: Cessation of CCL2 inhibition accelerates breast cancer metastasis by promoting angiogenesis. Nature 515: 130-133, 2014.

11. Tsai JH and Yang J: Epithelial-mesenchymal plasticity in carcinoma metastasis. Genes Dev 27: 2192-2206, 2013.

12. Turajlic $\mathrm{S}$ and Swanton $\mathrm{C}$ : Metastasis as an evolutionary process Science 352: 169-175, 2016

13. White BD, Chien AJ and Dawson DW: Dysregulation of Wnt/ $\beta$-catenin signaling in gastrointestinal cancers. Gastroenterology 142: 219-232, 2012.

14. Gu Y, Wang Q, Guo K, Qin W, Liao W, Wang S, Ding Y and Lin J: TUSC3 promotes colorectal cancer progression and epithelial-mesenchymal transition (EMT) through WNT/ $\beta$-catenin and MAPK signalling. J Pathol 239: 60-71, 2016.

15. Han B, Wang J, Gao J, Feng S, Zhu Y, Li X, Xiao T, Qi J and Cui W: DJ-1 as a potential biomarker for the early diagnosis in lung cancer patients. Tumour Biol 39: 1010428317714625, 2017.

16. Kawate T, Iwaya K, Koshikawa K, Moriya T, Yamasaki T, Hasegawa S, Kaise H, Fujita T, Matsuo H, Nakamura T, et al: High levels of DJ-1 protein and isoelectric point 6.3 isoform in sera of breast cancer patients. Cancer Sci 106: 938-943, 2015.

17. Benati M, Montagnana M, Danese E, Paviati E, Giudici S, Ruzzenente O, Franchi M and Lippi G: The clinical significance of DJ-1 and HE4 in patients with endometrial cancer. J Clin Lab Anal: 32, 2018 doi: 10.1002/jcla.22223.

18. Chan JY and Chan SH: Activation of endogenous antioxidants as a common therapeutic strategy against cancer, neurodegeneration and cardiovascular diseases: A lesson learnt from DJ-1. Pharmacol Ther 156: 69-74, 2015.

19. Yuen HF, Chan YP, Law S, Srivastava G, El-Tanani M, Mak TW and Chan KW: DJ-1 could predict worse prognosis in esophageal squamous cell carcinoma. Cancer Epidemiol Biomarkers Prev 17: 3593-3602, 2008

20. Jue C, Lin C, Zhisheng Z, Yayun Q, Feng J, Min Z, Haibo W, Youyang S, Hisamitsu T, Shintaro I, et al: Notch1 promotes vasculogenic mimicry in hepatocellular carcinoma by inducing EMT signaling. Oncotarget 8: 2501-2513, 2017.

21. Da C, Liu Y, Zhan Y, Liu K and Wang R: Nobiletin inhibits epithelial-mesenchymal transition of human non-small cell lung cancer cells by antagonizing the TGF- $\beta 1 / \mathrm{Smad} 3$ signaling pathway. Oncol Rep 35: 2767-2774, 2016.

22. Geng XF, Fang M, Liu SP and Li Y: Quantum dot-based molecular imaging of cancer cell growth using a clone formation assay. Mol Med Rep 14: 3007-3012, 2016.

23. Xiao J, Yang W, Xu B, Zhu H, Zou J, Su C, Rong J, Wang T and Chen Z: Expression of fibronectin in esophageal squamous cell carcinoma and its role in migration. BMC Cancer 18: 976 , 2018.

24. Zhu Y, Liu Y, Qian Y, Dai X, Yang L, Chen J, Guo S and Hisamitsu T: Research on the efficacy of Celastrus Orbiculatus in suppressing TGF- $\beta 1$-induced epithelial-mesenchymal transition by inhibiting HSP27 and TNF- $\alpha$-induced NF- $\kappa \mathrm{B} /$ Snail signaling pathway in human gastric adenocarcinoma. BMC Complement Altern Med 14: 433, 2014

25. Aleyasin H, Rousseaux MW, Marcogliese PC, Hewitt SJ Irrcher I, Joselin AP, Parsanejad M, Kim RH, Rizzu P, Callaghan SM, et al: DJ-1 protects the nigrostriatal axis from the neurotoxin MPTP by modulation of the AKT pathway. Proc Natl Acad Sci USA 107: 3186-3191, 2010.

26. Kim RH, Peters M, Jang Y, Shi W, Pintilie M, Fletcher GC, DeLuca C, Liepa J, Zhou L, Snow B, et al: DJ-1, a novel regulator of the tumor suppressor PTEN. Cancer Cell 7: 263-273, 2005.

27. Clements CM, McNally RS, Conti BJ, Mak TW and Ting JP: DJ-1, a cancer- and Pa2rkinson's disease-associated protein, stabilizes the antioxidant transcriptional master regulator Nrf2. Proc Natl Acad Sci USA 103: 15091-15096, 2006.

28. Hurst DR and Welch DR: Metastasis suppressor genes at the interface between the environment and tumor cell growth. Int Rev Cell Mol Biol 286: 107-180, 2011. 
29. Valastyan S and Weinberg RA: Tumor metastasis: Molecular insights and evolving paradigms. Cell 147: 275-292, 2011.

30. Clevers H: Wnt/beta-catenin signaling in development and disease. Cell 127: 469-480, 2006.

31. Nusse $\mathrm{R}$ and Clevers $\mathrm{H}$ : Wnt/ $\beta$-catenin signaling, disease, and emerging therapeutic modalities. Cell 169: 985-999, 2017.

32. Zhou J, Liu H, Zhang L, Liu X, Zhang C, Wang Y, He Q, Zhang Y, Li Y, Chen Q, et al: DJ-1 promotes colorectal cancer progression through activating PLAGL2/Wnt/BMP4 axis. Cell Death Dis 9: $865,2018$.

33. MacDonald BT, Tamai $\mathrm{K}$ and He X: Wnt/beta-catenin signaling: Components, mechanisms, and diseases. Dev Cell 17: 9-26, 2009.

34. Du C, Lv Z, Cao L, Ding C, Gyabaah OA, Xie H, Zhou L, Wu J and Zheng S: MiR-126-3p suppresses tumor metastasis and angiogenesis of hepatocellular carcinoma by targeting LRP6 and PIK3R2. J Transl Med 12: 259, 2014.

35. Tung EK, Wong BY, Yau TO and Ng IO: Upregulation of the Wnt Co-receptor LRP6 promotes hepatocarcinogenesis and enhances cell invasion. PLoS One 7: e36565, 2012.

36. Yamamoto $\mathrm{H}, \mathrm{Komekado} \mathrm{H}$ and Kikuchi A: Caveolin is necessary for Wnt-3a-dependent internalization of LRP6 and accumulation of beta-catenin. Dev Cell 11: 213-223, 2006.
37. Tang X, Fan Z, Wang Y, Ji G, Wang M, Lin J and Huang S: Expression of klotho and $\beta$-catenin in esophageal squamous cell carcinoma, and their clinicopathological and prognostic significance. Dis Esophagus 29: 207-214, 2016.

38. Chiurillo MA: Role of the Wnt/ $\beta$-catenin pathway in gastric cancer: An in-depth literature review. World J Exp Med 5: 84-102, 2015.

39. To SKY, Mak ASC, Eva Fung YM, Che CM, Li SS, Deng W, Ru B, Zhang J and Wong AST: $\beta$-catenin downregulates Dicer to promote ovarian cancer metastasis. Oncogene 36: 5927-5938, 2017.

40. Wu X, Luo F, Li J, Zhong X and Liu K: Tankyrase 1 inhibitior XAV939 increases chemosensitivity in colon cancer cell lines via inhibition of the Wnt signaling pathway. Int J Oncol 48: 1333-1340, 2016.

41. Guo W, Shen F, Xiao W, Chen J and Pan F: Wnt inhibitor XAV939 suppresses the viability of small cell lung cancer NCI-H446 cells and induces apoptosis. Oncol Lett 14: 6585-6591, 2017.

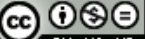

This work is licensed under a Creative Commons Attribution-NonCommercial-NoDerivatives 4.0 International (CC BY-NC-ND 4.0) License. 